\title{
DIFFUSIVE REALIZATIONS FOR SOLUTIONS OF SOME OPERATOR EQUATIONS: THE ONE-DIMENSIONAL CASE
}

\author{
MICHEL LENCZNER, GÉRARD MONTSENY, AND YOUSSEF YAKOUBI
}

\begin{abstract}
In this paper we deal with the derivation of state-realizations of linear operators that are solutions to certain operator linear differential equations in one-dimensional bounded domains. We develop two approaches in the framework of diffusive representations: one with complex diffusive symbols; the other with real diffusive symbols. Then, we illustrate the theories and develop numerical methods for a Lyapunov equation arising from optimal control theory of the heat equation. A practical purpose of this approach is real-time computation on a semi-decentralized architecture with low granularity.
\end{abstract}

\section{INTRODUCTION}

We consider an operator $u \mapsto z=P u$ a solution to an operator linear differential equation in a one-dimensional domain. An example, detailed in this paper, is the solution $P$ to a Lyapunov equation, arising from optimal control theory for the heat equation. A slightly simplified version reads as

$$
-\frac{d^{2}}{d x^{2}} P u-P \frac{d^{2}}{d x^{2}} u=Q u \text { for all } u \in H_{0}^{1}(0,1),
$$

$Q$ being a given operator. Our method is both fast and suitable for implementation with semi-decentralized computing architectures. Its formulation relates to an interesting technique applicable to causal operator realizations which is well developed in the context of time operators. One of the main advantages of this approach is its low computational cost; see the papers by G. Montseny et al. [16], 24], [13, [2], [3] and by D. Matignon et al. [10, 11, 22] for representations of various pseudodifferential operators and their approximation. C. Lubich et al. [21], 20], 17], [5], 26], [18] apply a similar idea to convolution operators and develop optimized numerical methods. They have reported detailed performance analysis of their methods in comparison with a direct approach via quadratures. Recently, C. Casenave and E. Montseny have shown in [4 how this approach yields stable schemes for integro-differential equations. G. Montseny has also published a monograph 25] on the subject in which the general framework, called diffusive representation, has been widely developed, and in which applications covering a wide range of fields were presented. Throughout our paper we adopt the same terminology. To date, many studies have focused on one-dimensional problems. We stress that in many cases, the theory of diffusive representation can be extended to any dimension. A

Received by the editor September 24, 2009 and, in revised form, September 23, 2010.

2010 Mathematics Subject Classification. Primary 35-xx, 47A62, 01-08, 47G10.

Key words and phrases. Integral operators, diffusive representation, operational equation, Lyapunov equation, computational method, real-time computation.

(C)2011 American Mathematical Society Reverts to public domain 28 years from publication 
higher-dimensional example, applied to image processing, can be found in 25]. So, it turns out that the theory presented in our paper can be extended to operator partial differential equations posed in multi-dimensional domains. We also mention that part of the results presented here was announced in [14].

All the previous works on diffusive representation deal with analytically known operators. This paper is new in that it considers operators that are not explicitly known, but are solutions to operator equations.

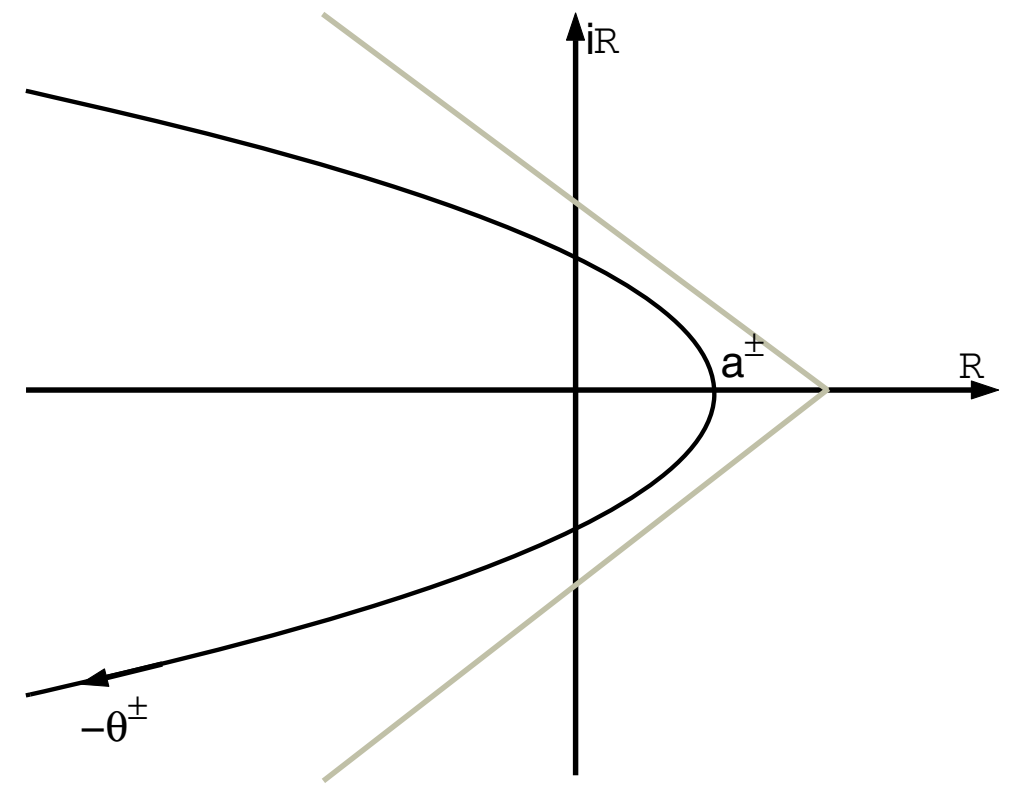

Figure 1. A path $-\theta^{ \pm}$

We consider the kernel decomposition of the realization of such an operator,

$$
P u(x)=\int_{0}^{1} p(x, y) u(y) d y
$$

and the decomposition $z=z^{+}+z^{-}$into causal and anti-causal parts,

$$
z^{+}(x)=\int_{0}^{x} p(x, y) u(y) d y \text { and } z^{-}(x)=\int_{x}^{1} p(x, y) u(y) d y .
$$

Thus, the diffusive realization of $P$ is split into two parts which read as

$$
z^{+}(x)=\int \mu^{+}(x, \xi) \psi^{+}(x, \xi) d \xi \text { and } z^{-}(x)=\int \mu^{-}(x, \xi) \psi^{-}(x, \xi) d \xi,
$$

where both $\psi^{+}$and $\psi^{-}$store a part of the history of the input $u$. They are respectively solutions to a forward and to a backward ordinary differential equation in $x$,

$$
\begin{aligned}
& \partial_{x} \psi^{+}(x, \xi)+\theta^{+}(\xi) \psi^{+}(x, \xi)=u(x) \text { with } \psi^{+}(0, \xi)=0, \\
& \partial_{x} \psi^{-}(x, \xi)-\theta^{-}(\xi) \psi^{-}(x, \xi)=u(x) \text { with } \psi^{-}(1, \xi)=0,
\end{aligned}
$$

where $\xi$ is a real parameter. We observe that they are defined independently of $P$. By contrast, the coefficients $\mu^{ \pm}$, called diffusive symbols, are dependent on $P$ but not 
on $u$. The functions $\xi \mapsto \theta^{+}(\xi)$ and $\theta^{-}(\xi)$ parameterize two closed paths in the complex plane; see Figure 1. The usual choices are parabolas, $-\theta^{ \pm}(\xi)=\theta_{p}^{ \pm}(i \xi+1)^{2}$ or hyperbolas, $-\theta^{ \pm}(\xi)=\theta_{h}^{ \pm}\left(1+\sin \left(i \xi-\alpha^{ \pm}\right)\right)$for $\xi \in \mathbb{R}$. Two different choices of $\mu^{ \pm}$are considered in this paper. To distinguish between them, we refer to them as complex diffusive symbols and real diffusive symbols to indicate that they are respectively complex and real-valued functions.

To build complex diffusive symbols we start by defining the kernels $p^{+}$and $p^{-}$ of the causal and anti-causal parts of the operator $P$, i.e., the restrictions of $p$ to $y<x$ and to $y>x$. We assume that the two functions

$$
y \mapsto \widetilde{p}^{ \pm}(x, y)=p^{ \pm}(x, x \mp y)
$$

are analytic and that their analytic extensions to $\mathbb{R}^{+}=[0,+\infty)$ admits Laplace transforms $\mathcal{P}^{ \pm}$. We also assume that $\mathcal{P}^{ \pm}$admits holomorphic extensions, vanishing towards infinity, from $\mathbb{C}^{+}=\mathbb{R}^{+}+i \mathbb{R}$ to the domain bounded to the left by $-\theta^{ \pm}$. Then, applying the Jordan lemma and the Cauchy theorem to the inverse Laplace transform $\mathcal{L}^{-1}$, the right-hand side relation $\widetilde{p}^{ \pm}=\mathcal{L}^{-1} \mathcal{P}^{ \pm}$is transformed into an integral along $-\theta^{ \pm}$. This yields new expressions for the kernels $p^{ \pm}$, which after replacement in (1)-(2) and identification, provide formulas for the complex diffusive symbols $\mu^{ \pm}$associated with $P$ and $\theta^{ \pm}$:

$$
\mu^{ \pm}(x, \xi)=\mp \frac{\theta^{ \pm \prime}(\xi)}{2 i \pi} \mathcal{P}^{ \pm}\left(x,-\theta^{ \pm}(\xi)\right)
$$

As already mentioned, the kernels $p^{ \pm}$are generally not analytically known, and in this paper we consider them as the solutions to two uncoupled very general boundary value problems. The uncoupling condition is imposed for the sake of simplicity, but it is by no means necessary. In the example of the Lyapunov equation, $q^{ \pm}$denoting the kernels of the causal and anti-causal parts of $Q$, the kernels $p^{ \pm}$are solutions to

$$
\begin{aligned}
-\Delta p^{ \pm}(x, y) & =q^{ \pm}(x, y) \text { in }(0,1)^{2} \text { with } \pm(x-y)>0, \\
\left(-\partial_{x}+\partial_{y}\right) p^{ \pm}(x, x) & =0 \text { on }(0,1), \text { and } p^{ \pm}=0 \text { on the rest of the boundary. }
\end{aligned}
$$

To compute complex diffusive symbols, we may develop a numerical method that follows the derivation steps of $\mu^{ \pm}$. However, it is worth noticing that the assumptions on $\mathcal{P}^{ \pm}$require both a regularity condition and a growth condition on the extended impulse responses $y \mapsto \widetilde{p}^{ \pm}(., y)$, which may be not fulfilled. For instance, in our example $p^{ \pm}$is generally not analytic (when $q^{ \pm}$is not analytic) and may comprise exponential terms. From the point of view of numerical computation, another limitation in our theory is the need for analytic extension of the impulse responses. To the best of our knowledge, building it numerically is an extremely time-consuming procedure. To circumvent these problems, we introduce an approximation method which does not strictly follow the continuous case. It is designed to be practicable in many cases where growth assumptions, and to a certain extent regularity assumptions are not fulfilled. The method amounts to building approximations $y \mapsto \widetilde{p}^{N \pm}(., y)$ of the impulse responses $y \mapsto \widetilde{p}^{ \pm}(., y)$ in an $N$-sized Galerkin base with negative exponential in $y$ and polynomials in $x$. Calculating their analytic extensions, their Laplace transforms $\mathcal{P}^{N \pm}$, and the poles of $\mathcal{P}^{N \pm}$ is a trivial task. Since the poles belong to $\mathbb{Z}^{-}$, the choice of the contours $\theta^{ \pm}$is considerably simplified, and efficient methods, already developed by several authors (see the discussion below), can be used for the quadratures involved in 
approximating $P u$. We notice that, by construction, the analytic extensions of $\widetilde{p}^{N \pm}$ and $\mu^{N \pm}$ (the corresponding diffusive symbols) are not convergent. However, the approximate diffusive realizations $z^{N \pm}$ are convergent (this is the only important point) which is confirmed by numerical tests.

Symbols may also be numerically determined from symbol equations instead of from kernel equations. From this point of view, the kernel calculation is an intermediate step that can be skipped. We have established the symbol equations for $p^{ \pm}$ as the kernel solutions to the general uncoupled boundary value problems. They take the form of integro-differential equations, with derivatives in $x$ and integrals in $\xi$. In our example, we write

$$
\begin{gathered}
\pm \int_{\mathbb{R}}\left(\partial_{x x}^{2}-2 \theta^{ \pm} \partial_{x}+2 \theta^{ \pm 2}\right) \mu^{ \pm} e^{\mp \theta^{ \pm}(x-y)} d \xi=q(x, y) \text { in }(0,1)^{2} \text { with } \pm(x-y)>0 \\
\int_{\mathbb{R}}\left(\partial_{x} \mp 2 \theta^{ \pm}\right) \mu^{ \pm} d \xi=0 \text { in }(0,1) \\
\int_{\mathbb{R}} \mu^{+} e^{-\theta^{+} x} d \xi=0, \int_{\mathbb{R}} \mu^{-} e^{\theta^{-}(x-1)} d \xi=0 \text { in }(0,1) \\
\int_{\mathbb{R}} \mu^{+} e^{-\theta^{+}(1-y)} d \xi=0, \int_{\mathbb{R}} \mu^{-} e^{-\theta^{-} y} d \xi=0 \text { on the other boundaries. }
\end{gathered}
$$

As already mentioned, even when the exact symbols do not exist, approximate symbols $\mu^{N \pm}$ may be built. Those are solutions to unsymmetrical variational formulations discretized by a Petrov-Galerkin method whose bases are inspired from that used in the Galerkin method for $p^{N \pm}$.

In the special case where all singularities of $\mathcal{P}^{ \pm}$lie on the negative real axis, the paths $\theta^{ \pm}$can be so degenerate that they can be confined to the real axis. The construction of complex diffusive symbols is still applicable (apart from further mathematical technicalities, see [25]). We can also use an alternative diffusive symbol derivation yielding $\mu^{ \pm *}$, the so-called real diffusive symbols. By this method, the partial functions $\xi \mapsto \mu^{ \pm *}(., \xi)$ are defined as the images through $\mathcal{L}^{-1}$ of the analytic extension of $y \mapsto \widetilde{p}^{ \pm}(., y)$. As for complex diffusive symbols, we find the integro-differential equations satisfied by real symbols. To find their solution, we suggest a computational method based on a decomposition of the solution into a regular part and singular terms, which, in the example, reads as

$$
\mu^{ \pm *}(x, \xi)=g^{ \pm}(x, \xi)+\sum_{n=0}^{n_{0}} g_{n}^{ \pm}(x) \delta^{(n)}(\xi) .
$$

Once the diffusive symbols are available, another concern is the numerical approximation of the integrals in (2). The diffusive realization $z(x)=z^{+}(x)+z^{-}(x)$ being evaluated at some points $\left(x_{n}\right)_{n=0, \ldots, \mathcal{N}} \in(0,1)$, the corresponding values $z\left(x_{n}\right)$ are evaluated thanks to a quadrature rule by

$$
z_{n}=\sum_{k} \mu_{n, k}^{+} \psi_{n, k}^{+}+\mu_{n, k}^{-} \psi_{n, k}^{-}
$$

where $\mu_{n, k}$ is the discrete $\mu$ at the point $\left(x_{n}, \xi_{k}\right)$, and $\psi_{n, k}$ is a discrete approximation of $\psi$ at $\left(x_{n}, \xi_{k}\right)$. Such a quadrature rule is similar to those encountered in an inverse Laplace transform. This method has been extensively studied. The approach followed in this paper is to apply a simple trapezoid rule. Optimal parameters for three classes of contours (parabolas, hyperbolas, and cotangent contours) 
have been derived. The latter, also referred to as Talbot contours, was first studied by A. Talbot in a paper [29] published in 1979, and was recently revisited by L.N. Trefethen and J.A.C. Weideman in [30]. Parabolas have been investigated by I.P. Gavrilyuk and V.L. Makarov in 77. The case of hyperbolic contours, a particularly attractive choice for generalizations to sectorial operators, has been treated by many authors [17, 19, 23, 28, and [8. Our computations of the integrals in (2) are based on optimized paths and quadrature rules that we will detail in a forthcoming paper.

As we have already mentioned, another feature of the diffusive realization method, when applied to spatially distributed operators, is that it yields to real-time computations implementable on semi-decentralized architectures with low granularity such as FPGAs; see 9 . To justify this point, we consider that the data in the previous algorithm are allocated in a set of $\mathcal{N}+1$ processing units corresponding to the nodes $\left(x_{n}\right)_{n=0, \ldots, \mathcal{N}}$. Since a computation is said to be suited for a semi-decentralized architecture when required communications are only between close neighbors, an algorithm is considered as appropriate for such architecture if operations are between functions evaluated at $x_{n}$ and at its neighbors $x_{n-1}$ and $x_{n+1}$. In a real-time computation, for a time dependent flow $\left(u_{n}\right)_{n}$ of spatially discrete inputs, the diffusive symbols are computed in a preprocessing step and only the computation of $\psi_{n, k}^{ \pm}$, and then of $z_{n}$ through (6), remain to be done at each time step. The summation (6) is clearly a local (in space) operation, and the resolution of the Cauchy problems (3)-(4) requires operations between neighboring nodes only. Actually, considering the recurrence relation associated with a forward discretization method (the same would be true for a backward discretization method),

$$
\psi_{n+1, k}^{+}=\alpha_{k}^{+} \psi_{n, k}^{+}+\beta_{k}^{+} u_{n}+\gamma_{k}^{+} u_{n+1}, \quad \psi_{0, k}^{+}=0,
$$

the approximation $\psi_{n+1, k}^{+}$at a node $x_{n+1}$ is derived from the approximation $\psi_{n, k}^{+}$ at the previous node $x_{n}$ for all $k$.

Now, we comment on the advantage of our approach compared to other works [1], 12], 15] on the problem of realizations, on semi-decentralized architectures, of operators issued from optimal control for partial differential equations. Such operators are solutions to Riccati equations, which are nonlinear, and therefore do not fall within the scope of our paper. However, the theories are sufficiently general to apply to linear operational equations as well. The paper [1 of B. Bamieh's group focuses on partial differential equations posed in the whole space $\mathbb{R}^{d}$ with controls and observations distributed over the entire space. The paper [6] covers a very restricted class of boundary value problems with observations and controls distributed over the entire space also. The method used in [15] is applicable to a much broader class of boundary value problems posed in bounded or unbounded domains, but with the same restriction on control and observation operators. In the present paper, the domain is bounded and the operators are not assumed to be distributed over the whole domain. The main limitations are that the operational equations are linear, posed in a one-dimensional domain and the resulting boundary value problems solved by $p^{+}$and $p^{-}$must be uncoupled. These three assumptions have been introduced for the sake of simplicity, but none of them is an obstacle to further generalizations.

The paper is organized as follows. The framework of diffusive realizations of integral operators is presented in Section 2. In Section 3, we establish the equations 
satisfied by complex and real diffusive symbols. Section 4 describes an application to the Lyapunov Equation. Section 5 is about numerical methods. Finally, a technical lemma used for real symbol calculation is reported in an Appendix in Section 6.

\section{DifFUSIVE REALizATION OF INTEGRAL OPERATORS}

We consider bounded operators $P$ in a subset of $L^{2}(\omega)$ formulated in the general integral form

$$
P u(x)=\int_{\omega} p(x, y) u(y) d y,
$$

where $\omega=] 0,1[$, and where the regularity of the kernel $p(x, y)$ will be specified later 1

2.1. Definition and general properties of diffusive realization. An operator $P$ is said to be causal (respectively anti-causal) if $p(x, y)=0$ for $y>x$ (respectively for $y<x$ ). Diffusive realizations of $P$ are based on its (unique) decomposition into causal and anti-causal parts,

$$
P=P^{+}+P^{-}
$$

where

$$
P^{+} u(x)=\int_{0}^{x} p(x, y) u(y) d y \text { and } P^{-} u(x)=\int_{x}^{1} p(x, y) u(y) d y .
$$

Throughout this paper, we shall use the superscripts + or - to refer to causal or anti-causal operators, and the convention $\mp=-( \pm)$.

The so-called impulse response $\widetilde{p}$ is defined from the kernel $p(x, y)$ by

$$
p(x, y)=\widetilde{p}(x, x-y) \text { with }(x, y) \in \bar{\Omega}
$$

or conversely by

$$
\widetilde{p}(x, y)=p(x, x-y) \text { with }(x, x-y) \in \bar{\Omega}
$$

where $\Omega=\omega \times \omega$.

For given $a^{ \pm} \in \mathbb{R}$, we consider $\xi \mapsto \theta^{ \pm}(\xi)$ two-complex continuous and almost everywhere differentiable functions from $\mathbb{R}$ to $\left[a^{ \pm},+\infty[+i \mathbb{R} \subset \mathbb{C}\right.$, with derivatives $\theta^{ \pm \prime}$ such that $0<\alpha \leq\left|\theta^{ \pm \prime}\right| \leq \beta<+\infty$, and which define two simple oriented arcs closed at infinity. We also suppose that $\theta^{ \pm}$are located inside a sector defined by two nonvertical straight lines as shown in Figure 1. Note that this last condition implies that the forthcoming equation (13, ) is of a diffusive nature (see 33]); this justifies the terminology "diffusive representation". In such a case, the associated semigroup is analytic with as a consequence that the impulse response of the operator will be analytic in $\mathbb{R}^{+*}=\mathbb{R}^{+}-\{0\}$ and in $\mathbb{R}^{*-}=\mathbb{R}^{-}-\{0\}$ with $\mathbb{R}^{-}=(-\infty, 0]$.

Remark 1. The approach presented hereafter may also be formulated with bounded arcs $\theta^{ \pm}$parameterized on $\mathbb{R} / 2 \pi \mathbb{R} \equiv\left[0,2 \pi\right.$ [ instead of $\mathbb{R}$, so that $\theta^{ \pm}$are closed contours. Apart from minor technical adaptations, all of the results of this section remain valid after changing $\mathbb{R}$ into $[0,2 \pi[$.

\footnotetext{
${ }^{1}$ Note that for the applications we have in mind, unbounded operators may occur; in such a case, they can be decomposed as products of a differential operator and an operator belonging to the class considered here.
} 
From now on, we use the convenient notation:

$$
\langle\mu, \psi\rangle:=\int \mu(\xi) \psi(\xi) d \xi
$$

where the integration set is clear from the context in each particular instance. Note that when $\mu$ is not a locally integrable function, a more general duality product, to be specified in each concrete cast2, is involved in place of the integral.

Definition 2. (i) A causal operator $P^{+}$(resp. anti-causal operator $P^{-}$) admits a $\theta^{+}$-diffusive realization (resp. $\theta^{-}$-diffusive realization) if there exists a so-called diffusive symbo $\mu^{3} \mu^{+}(x, \xi)$ (resp. $\left.\mu^{-}(x, \xi)\right)$ such that

$$
\left.P^{+} u(x)=\left\langle\mu^{+}, \psi^{+}(u)\right\rangle \text { (resp. } P^{-} u(x)=\left\langle\mu^{-}, \psi^{-}(u)\right\rangle\right),
$$

where $\psi^{ \pm}$, then the so-called $\theta^{ \pm}$-representations of $u$, are defined by

$$
\begin{aligned}
& \psi^{+}(u)(x, \xi)=\int_{0}^{x} e^{-\theta^{+}(\xi)(x-y)} u(y) d y, \\
& \psi^{-}(u)(x, \xi)=-\int_{x}^{1} e^{\theta^{-}(\xi)(x-y)} u(y) d y \forall \xi \in \mathbb{R} .
\end{aligned}
$$

(ii) An operator $P$ admits a $\theta^{ \pm}$-diffusive realization if both $P^{+}$and $P^{-}$, its causal and anti-causal parts, admit a diffusive realization associated respectively with $\theta^{+}$and $\theta^{-}$.

Note that in (11), $u$ can be taken in the space of measures without loss of regularity of $\psi$; therefore, the causal part of the impulse response can be written as

$$
\widetilde{p}(x, y)=\left\langle\mu(x, \xi), e^{-\theta^{+}(\xi) y}\right\rangle,
$$

and it ensures that the Fubini theorem is valid:

$$
\int_{0}^{x}\left\langle\mu, e^{-\theta^{+}(\xi)(x-y)}\right\rangle u(y) d y=\left\langle\mu, \int_{0}^{x} e^{-\theta^{+}(\xi)(x-y)} u(y) d y\right\rangle .
$$

The same thing can be done for the anti-causal part.

The functions $\psi^{ \pm}(u)$ can be characterized as the unique solutions to the following direct and backward Cauchy problems, parameterized by $\xi \in \mathbb{R}$ :

$$
\begin{aligned}
& \left.\partial_{x} \psi^{+}(x, \xi)=-\theta^{+}(\xi) \psi^{+}(x, \xi)+u(x) \forall x \in\right] 0,1\left[, \psi^{+}(0, \xi)=0,\right. \\
& \left.\partial_{x} \psi^{-}(x, \xi)=\theta^{-}(\xi) \psi^{-}(x, \xi)+u(x) \forall x \in\right] 0,1\left[, \psi^{-}(1, \xi)=0 .\right.
\end{aligned}
$$

Together with (11), they constitute a diffusive state-space realization of $P^{+}$and $P^{-}$respectively. This last point is central in view of the concrete approximated realizations of $P$.

The following proposition states that symmetric operators can be expressed with respect to $\mu^{+}$or $\mu^{-}$only. This property may be useful for instance in the treatment of nonlinear operator equations such as Riccati equations, where the equations of $\mu^{+}$and $\mu^{-}$are coupled.

${ }^{2}$ Note that a duality fitted to the general framework of diffusive realization has been introduced in 25 .

${ }^{3} \mathrm{Up}$ to the function $\theta, \xi$ is indeed a frequency variable because homogeneous to $1 / x$. 
Proposition 3. If there exists a diffusive realization of a symmetric operator $P$, then it may be realized with only one of the two symbols $\mu^{+}$or $\mu^{-}$:

$$
\begin{aligned}
& P u(x)=\left\langle\mu^{+}(x, \xi), \psi^{+}(u)(x, \xi)\right\rangle+\int_{x}^{1}\left\langle\mu^{+}(y, \xi), e^{-\theta^{+}(\xi)(y-x)}\right\rangle u(y) d y, \\
& P u(x)=-\int_{0}^{x}\left\langle\mu^{-}(y, \xi), e^{\theta^{-}(\xi)(y-x)}\right\rangle u(y) d y-\left\langle\mu^{-}(x, \xi), \psi^{-}(u)(x, \xi)\right\rangle .
\end{aligned}
$$

The relations between the causal and anti-causal parts of the kernel and the diffusive symbols are then

$$
\begin{aligned}
& p(x, y)=\left\langle\mu^{+}(x, \xi), e^{-\theta^{+}(\xi)(x-y)}\right\rangle=-\left\langle\mu^{-}(y, \xi), e^{\theta^{-}(\xi)(y-x)}\right\rangle \text { for } y \leq x, \\
& p(x, y)=-\left\langle\mu^{-}(x, \xi), e^{\theta^{-}(\xi)(x-y)}\right\rangle=\left\langle\mu^{+}(y, \xi), e^{-\theta^{+}(\xi)(y-x)}\right\rangle \text { for } y \geq x .
\end{aligned}
$$

Proof. According to the expression of diffusive realizations of $P^{+}$and $P^{-}$, one deduces the relation between $p(x, y)$ and $\mu^{ \pm}$,

$$
\begin{array}{ll}
p(x, y)=\left\langle\mu^{+}(x, \xi), e^{-\theta^{+}(\xi)(x-y)}\right\rangle & \text { for } y \leq x, \\
p(x, y)=-\left\langle\mu^{-}(x, \xi), e^{\theta^{-}(\xi)(x-y)}\right\rangle & \text { for } x \leq y .
\end{array}
$$

Now, the symmetry condition $p(x, y)=p(y, x)$ yields an expression of

$$
P^{-} u(x)=\int_{x}^{1} p(y, x) u(y) d y,
$$

with a kernel $p(y, x)$ for $x<y$ that may be formulated as a function of $\mu^{+}$, so that the first formula for $P u$ follows. The second one is obtained using a similar argument that leads to an expression of $P^{+} u$ with respect to $\mu^{-}$.

2.2. Complex diffusive realization. In this subsection we state sufficient conditions for the existence of $\theta^{ \pm}$-diffusive realizations with complex diffusive symbols $\mu^{ \pm}$when $\theta^{+}$and $\theta^{-}$are some given paths. The derivation starts from the impulse response and is constructive.

Existence conditions for complex symbols pertain to

$$
\mathcal{P}^{+}(x, \lambda)=\mathcal{L}_{y}(\widetilde{p}(x, y))(\lambda) \text { and } \mathcal{P}^{-}(x, \lambda)=\mathcal{L}_{y}(\widetilde{p}(x,-y))(\lambda),
$$

the Laplace transforms, with respect to $y$, of the extended causal and anti-causal parts of the impulse response (assumed to be locally integrable).

Assumption 4. (i) The Laplace transform $\lambda \mapsto \mathcal{P}^{+}(x, \lambda)\left(\right.$ resp. $\left.\lambda \mapsto \mathcal{P}^{-}(x, \lambda)\right)$ is holomorphic in a domain $D^{+} \subset \mathbb{C}\left(\right.$ resp. $\left.D^{-} \subset \mathbb{C}\right)$ that contains the closed set located to the right of the arc $-\theta^{+}\left(\right.$resp. $\left.-\theta^{-}\right)$;

(ii) For each $x \in \omega$, the Laplace transform $\lambda \mapsto \mathcal{P}^{+}(x, \lambda)\left(\right.$ resp. $\left.\lambda \mapsto \mathcal{P}^{-}(x, \lambda)\right)$ vanishes uniformly with respect to $\arg \lambda$ when $|\lambda| \rightarrow \infty$.

As a matter of fact, there exists a relation between the holomorphy of a function in a sector including $\mathbb{R}^{+}$and the holomorphy of its Laplace transform extended to a sector with an angle larger than $\pi$. We state it in Theorem 7 , which results from Theorems 1.46.5 and 1.46.6 of 31. For any $\alpha, \beta \in \mathbb{R}$, we define the sector $\Sigma_{\alpha \beta}=\left\{y \in \mathbb{C}^{*} \mid \arg (y) \in(\alpha, \beta)\right\}$ and the half-line $\mathcal{D}_{t}=\left\{r e^{i t} \mid r>0\right\}$. 
Assumption 5. There exist some constants,

$$
-\frac{\pi}{2}<\alpha^{ \pm}<0<\beta^{ \pm}<\frac{\pi}{2}, a^{ \pm}, A^{ \pm}>0, \rho^{ \pm} \geq 0 \text { and } \tau^{ \pm}>-1,
$$

such that for each $x \in \omega, y \mapsto \widetilde{p}(x, \pm y)$ is holomorphic in $\Sigma_{\alpha^{ \pm} \beta^{ \pm}}$. Moreover, for all $y \in \Sigma_{\alpha^{ \pm} \beta^{ \pm}}$

$$
|\widetilde{p}(x, \pm y)| \leq c|y|^{\tau^{ \pm}} \text {when }|y| \leq a^{ \pm} \text {and }|\widetilde{p}(x, y)| \leq c e^{\tau^{ \pm}}|y| \text { when }|y| \geq A^{ \pm},
$$

for some positive constants $c$.

Assumption 6. For all $t \in(\alpha, \beta)$,

$$
\widetilde{p}(x, \pm y) \sim \sum_{j=0}^{\infty} a_{j}^{ \pm}( \pm y)^{\gamma_{j}^{ \pm}} \text {when } y \rightarrow 0 \text { with } \pm y \in \mathcal{D}_{t}
$$

for two sequences

$$
-1<\operatorname{Re} \gamma_{0}^{ \pm}<\operatorname{Re} \gamma_{1}^{ \pm}<\cdots<\operatorname{Re} \gamma_{j}^{ \pm}<\operatorname{Re} \gamma_{j+1}^{ \pm}<\cdots \text { with } \lim _{j \rightarrow \infty} \operatorname{Re} \gamma_{j}^{ \pm}=\infty .
$$

Theorem 7. Under Assumption [5 the Laplace transforms $\lambda \mapsto \mathcal{P}^{ \pm}(., \lambda)$ are extended into a holomorphic function in the sector $\Sigma_{\alpha^{ \pm}-\frac{\pi}{2}, \beta^{ \pm}+\frac{\pi}{2}}$. Moreover, if Assumption 6 is fulfilled, the extensions $\mathcal{P}^{ \pm}$admit, for all $\varepsilon>0$, an asymptotic expansion

$$
\mathcal{P}^{ \pm}(., \lambda)=\sum_{j=0}^{\infty} a_{j}^{ \pm} \Gamma\left(\gamma_{j}^{ \pm}+1\right) \lambda^{-\left(\gamma_{j}^{ \pm}+1\right)}
$$

when $\lambda \rightarrow \infty$ and $\lambda \in \Sigma_{\alpha^{ \pm}-\frac{\pi}{2}+\varepsilon, \beta^{ \pm}+\frac{\pi}{2}-\varepsilon}$.

Next, we state the theorem of existence of a complex $\theta^{ \pm}$-diffusive realization with complex diffusive symbols.

Theorem 8. For a given path $\theta^{+}$(resp. $\left.\theta^{-}\right)$, if a causal (resp. anti-causal) operator $P^{+}$(resp. $\left.P^{-}\right)$satisfies Assumptions 4, then there exists a complex $\theta^{+}$-diffusive symbol $\mu^{+}$(resp. $\theta^{-}$-diffusive symbol $\left.\mu^{-}\right)$,

(17) $\mu^{+}(x, \xi)=-\frac{\theta^{+\prime}(\xi)}{2 i \pi} \mathcal{P}^{+}\left(x,-\theta^{+}(\xi)\right)\left(\operatorname{resp} . \mu^{-}(x, \xi)=\frac{\theta^{-\prime}(\xi)}{2 i \pi} \mathcal{P}^{-}\left(x,-\theta^{-}(\xi)\right)\right)$, and $\xi \mapsto \mu^{+}(x, \xi)$ (resp. $\left.\xi \mapsto \mu^{-}(x, \xi)\right)$ has the same regularity as $\theta^{+\prime}$ (resp. $\theta^{-\prime}$ ).

Proof. The Laplace transform $\mathcal{P}^{+}$is holomorphic to the right half-plane $\operatorname{Re}(z) \geq a$, then $\widetilde{p}(x, y)$ can be expressed, thanks to the inverse $\mathcal{L}^{-1}$ of the Laplace transform by

$$
\widetilde{p}(x, y)=\mathcal{L}^{-1}\left(\mathcal{P}^{+}(x, \lambda)\right)(y)=\frac{1}{2 i \pi} \int_{a+i \mathbb{R}} \mathcal{P}^{+}(x, \lambda) e^{\lambda y} d \lambda .
$$

Since for all $x \in \omega$ the Laplace transform $\lambda \mapsto \mathcal{P}^{+}(x, \lambda)$ is assumed to be holomorphic on the right of $-\theta^{+}$and to vanish uniformly at infinity, the Jordan lemma and the Cauchy theorem allow us to prove that

$$
\widetilde{p}(x, y)=\frac{1}{2 i \pi} \int_{-\theta^{+}} \mathcal{P}^{+}(x, \lambda) e^{\lambda y} d \lambda=\int_{\mathbb{R}} \frac{-\theta^{+^{\prime}}(\xi)}{2 i \pi} \mathcal{P}^{+}\left(x,-\theta^{+}(\xi)\right) e^{-\theta^{+}(\xi) y} d \xi .
$$


To complete the proof of the causal part we use the relation $P^{+} u(x)=\int_{0}^{x} \tilde{p}(x, x-$ $y) u(y) d y$ and the expression (11) of $\psi^{+}$. The proof is similar for the anti-causal part. Considering $\widetilde{p}(x,-y)$ when $y<0$, we get

$$
\widetilde{p}(x,-y)=\frac{1}{2 i \pi} \int_{-\theta^{-}} \mathcal{P}^{-}(x, \lambda) e^{\lambda y} d \lambda=\int_{\mathbb{R}} \frac{-\theta^{-\prime}(\xi)}{2 i \pi} \mathcal{P}^{-}\left(x,-\theta^{-}(\xi)\right) e^{-\theta^{-}(\xi) y} d \xi
$$

and we conclude thanks to the expressions of $P^{-} u$ and of $\psi^{-}$.

Remark 9. Notice that according to our Assumption 4 the causal (resp. anticausal) part of the impulse response is necessarily analytic on $\mathbb{R}^{+*}$ (resp. $\mathbb{R}^{-*}$ ) and locally integrable on $\mathbb{R}^{+}$(resp. $\mathbb{R}^{-}$) with respect to the second variable $y$. As a matter of fact, this can be observed, for instance, on the causal part. The expression,

$$
\widetilde{p}(x, y)=\int_{\mathbb{R}} \frac{-\theta^{+\prime}(\xi)}{2 i \pi} \mathcal{P}^{+}\left(x,-\theta^{+}(\xi)\right) e^{-\theta^{+}(\xi) y} d \xi,
$$

may be extended to a thin strip constituting a vicinity of $\mathbb{R}^{+}$in $\mathbb{C}$. Thanks to our hypothesis on $\theta^{+}$this function is differentiable, thus it is analytic in the open strip and therefore in $\mathbb{R}^{+*}$.

Remark 10. When it exists, the complex diffusive symbol resulting from the above derivation is necessarily unique, but an infinity of other complex diffusive symbols exists also. This can be seen by inspection of the kernels of the linear operators

$$
\mu^{ \pm} \mapsto\left\langle\mu^{ \pm}, \psi^{ \pm}(u)\right\rangle,
$$

which include any function $\xi \mapsto \mu^{ \pm}(., \xi)$ defined by (17) with $\mathcal{P}^{+}$(resp. $\mathcal{P}^{-}$) holomorphic in the closure of the domain to the left of $-\theta^{+}$(resp. of $\left.-\theta^{-}\right)$.

The next proposition shows that half of $\theta^{ \pm}$is sufficient to realize real-valued operators.

Proposition 11. If $P$ is real-valued and if the paths $\theta^{ \pm}$are symmetric with respect to the real axis, that is, $\theta^{ \pm}(-\xi)=\overline{\theta^{ \pm}(\xi)}$, then $\mu^{ \pm}(-\xi)=\overline{\mu^{ \pm}(\xi)}$ and

$$
\left\langle\mu^{ \pm}, \psi^{ \pm}\right\rangle=2 \operatorname{Re} \int_{0}^{+\infty} \mu^{ \pm} \psi^{ \pm} d \xi
$$

so that the diffusive realization can be determined with a half path $\theta^{ \pm *}=\theta_{\mid \mathbb{R}^{+}}^{ \pm}$.

Proof. If $\theta^{ \pm}(-\xi)=\overline{\theta^{ \pm}(\xi)}$, then $\psi^{ \pm}(x,-\xi)=\overline{\psi^{ \pm}(x, \xi)}$. Since $P$ is real-valued, then $\mathcal{P}^{ \pm}(x, \bar{\lambda})=\overline{\mathcal{P}^{ \pm}(x, \lambda)}$ and $\mu^{ \pm}(-\xi)=\overline{\mu^{ \pm}(\xi)}$. Therefore, we have

$$
\begin{aligned}
\left\langle\mu^{ \pm}, \psi^{ \pm}\right\rangle & =\int_{0}^{+\infty} \mu^{ \pm} \psi^{ \pm} d \xi+\int_{-\infty}^{0} \mu^{ \pm} \psi^{ \pm} d \xi \\
& =\int_{0}^{+\infty} \mu^{ \pm}(x, \xi) \psi^{ \pm}(x, \xi)+\mu^{ \pm}(x,-\xi) \overline{\psi^{ \pm}(x, \xi)} d \xi
\end{aligned}
$$

So $\mu^{ \pm}(-\xi)=\overline{\mu^{ \pm}(\xi)}$ yields

$$
\left\langle\mu^{ \pm}, \psi^{ \pm}\right\rangle=2 \operatorname{Re} \int_{0}^{+\infty} \mu^{ \pm} \psi^{ \pm} d \xi
$$


2.3. Real diffusive realizations. In this section, we derive another formula for diffusive symbols when all singularities of the Laplace transforms $\mathcal{P}^{ \pm}$are located on the real axis $\mathbb{R}$, and when the contours $\theta^{ \pm}(\mathbb{R})$ are embedded in a semi-infinite line in $\mathbb{R}$. This special case is not covered by Theorem 8 where we have assumed that the paths $\theta^{ \pm}$do not intersect with the singularities of $\mathcal{P}^{ \pm}$. We refer to [25] for a more general framework allowing singularities on the paths $\theta^{ \pm}$. It yields to more technical proofs. It indeed requires considering $\int_{\theta}$ in the sense of right trace, and the resulting complex diffusive symbols $\mu^{ \pm}$as generalized functions. From the topological viewpoint, it involves appropriate Fréchet spaces $\Delta_{\theta^{ \pm}}$for the functions $\psi^{ \pm}$and their topological dual $\Delta_{\theta^{ \pm}}^{\prime}$ for diffusive symbols. Regarding uniqueness, Remark 10 still applies and proves that, for such a contour with a void interior, complex diffusive symbols are unique. Here we follow an alternative approach where an impulse response turns out to be the Laplace transform of a diffusive symbol.

We start with a general remark on diffusive realizations with closed paths $\theta^{ \pm}$ having an empty interior. They may be parameterized symmetrically if we choose $\theta^{ \pm}(-\xi)=\theta^{ \pm}(\xi)$. Thus $\psi^{ \pm}(-\xi)=\psi^{ \pm}(\xi)$, and

$$
\begin{aligned}
\left\langle\mu^{ \pm}, \psi^{ \pm}\right\rangle & =\int_{0}^{+\infty} \mu^{ \pm}(x, \xi) \psi^{ \pm}(x, \xi) d \xi+\int_{-\infty}^{0} \mu^{ \pm}(x, \xi) \psi^{ \pm}(x, \xi) d \xi \\
& =\int_{0}^{+\infty}\left(\mu^{ \pm}(x, \xi)+\mu^{ \pm}(x,-\xi)\right) \psi^{ \pm}(x, \xi) d \xi
\end{aligned}
$$

Posing $\mu^{ \pm *}(x, \xi)=\mu^{ \pm}(x, \xi)+\mu^{ \pm}(x,-\xi)$ yields a diffusive realization on the half paths $\theta^{ \pm *}=\theta_{\mid \mathbb{R}^{+}}^{ \pm}$(parameterized on $\mathbb{R}^{+}$only) formally expressed by

$$
=\int_{0}^{+\infty} \mu^{ \pm *}(x, \xi) \psi^{ \pm}(x, \xi) d \xi
$$

More rigorously, since $\mu^{ \pm *}$ can be generalized functions, the integral will preferably be denoted by a duality product $\left\langle\mu^{ \pm *}, \psi^{ \pm}\right\rangle$. An important particular case is when $\theta^{ \pm *}$ are straight half-lines,

$$
\theta^{ \pm *}(\xi)=\lambda_{0}^{ \pm}+\sigma^{ \pm} \xi
$$

with $\xi \in \mathbb{R}^{+}, \lambda_{0}^{ \pm}$and $\sigma^{ \pm}$being complex numbers; then

$$
\widetilde{p}(x, \pm y)= \pm e^{-\lambda_{0}^{ \pm} y}\left(\mathcal{L}_{\xi} \mu^{ \pm *}(x, \xi)\right)\left(\sigma^{ \pm} y\right) \text { for } y \in \mathbb{R}^{+},
$$

where $\mathcal{L}$ is the Laplace transform extended to the set $\mathcal{D}_{+}^{\prime}$ of distributions with support in $\mathbb{R}^{+}$. Equivalently the expressions of $\mu^{ \pm *}$ with respect to $\widetilde{p}$ are

$$
\mu^{ \pm *}(x, \xi)= \pm \mathcal{L}^{-1}\left(e^{\lambda_{0}^{ \pm} y / \sigma^{ \pm}} \widetilde{p}\left(x, \pm y / \sigma^{ \pm}\right)\right)(\xi) \text { for } \xi \in \mathbb{R}^{+} .
$$

As we consider real operators (and so real impulse responses) only, the singularities of $\mathcal{P}^{ \pm}$are necessarily conjugate. Accordingly, they are concentrated on a half-line embedded in the real axis. In the following, we restrict to parameters $\lambda_{0}^{ \pm}$and $\sigma^{ \pm} \in \mathbb{R}$, so $\mu^{ \pm *}$ is a real-valued function. It is called a real diffusive symbol, and the associated realization a real $\theta^{ \pm *}$-diffusive realization.

To close this section, we state a sufficient condition for such a diffusive realization to exist.

Assumption 12. (i) The function $y \mapsto \tilde{p}(., y)$ (resp. $y \mapsto \tilde{p}(.,-y)$ ) has an analytic continuation holomorphic in $\mathbb{R}^{+*}+i \mathbb{R}$. 
(ii) There exists a constant $c^{+} \in \mathbb{R}^{+}$(resp. $c^{-} \in \mathbb{R}^{+}$) such that $y \in \mathbb{C} \mapsto$ $\tilde{p}(x, y) e^{\lambda_{0}^{+} y}$ (resp. $\left.y \in \mathbb{C} \mapsto \tilde{p}^{-}(x, y) e^{\lambda_{0}^{-} y}\right)$ is bounded uniformly in $x$ by a polynomial function of $|y|$ in the half-plane Rey $>c^{+}\left(\right.$resp. $\left.>c^{-}\right)$.

Proposition 13. If Assumption 12 is fulfilled, then for straight half-lines $\theta^{ \pm *}$ as in (19), the operator $P$ admits a $\theta^{ \pm *}$-diffusive realization with unique symbols $\mu^{ \pm *} \in \mathcal{D}_{+}^{\prime}$.

Proof. This statement comes directly from the characterization of the range of $\mathcal{D}_{+}^{\prime}$ by the Laplace transform; see e.g. 27] Chap. VI, Proposition 5.

\section{Diffusive SYMBolic FORMULATION OF LINEAR OPERATIONAL EQUATIONS}

In this section we consider a kernel $p(x, y)$ solution to a general linear boundary value problem with variable coefficients. We introduce the integro-differential equations satisfied by its complex symbol $\mu^{ \pm}$and by its real symbol $\mu^{ \pm *}$ provided that they exist for some given paths $\theta^{ \pm}$and $\theta^{ \pm *}$.

We start by dividing $\Omega$ into two open sets $\Omega^{+}$and $\Omega^{-}$corresponding to the causal $(y<x)$ and anti-causal $(y>x)$ parts of $\Omega$. The boundary of $\Omega^{+}$is split into $\Gamma_{y}^{+}=\{1\} \times \omega, \Gamma_{x}^{+}=\omega \times\{0\}$ and $\Gamma_{0}=\{(x, y) \in \Omega$ s.t. $x=y\}$, when the boundary of $\Omega^{-}$is split into $\Gamma_{y}^{-}=\{0\} \times \omega, \Gamma_{x}^{-}=\omega \times\{1\}$ and $\Gamma_{0}$.

Denoting by $\nabla=\left(\partial_{x}, \partial_{y}\right)^{T}$ and by $q$ the kernel of a second operator $Q$, we consider that $p$ is the solution to a partial differential equation written in the general form,

$$
A(x, y, \nabla) p(x, y)=q(x, y) \text { in } \Omega^{+} \cup \Omega^{-},
$$

along with boundary conditions,

$$
B(x, y, \nabla) p(x, y)=r(x, y) \text { on } \partial \Omega^{+} \cup \partial \Omega^{-} .
$$

We introduce the change of variable $\phi^{ \pm}(x, y)=(x, x \mp y)$, the modified impulse responses

$$
\widetilde{p}^{ \pm}(x, y)=\widetilde{p}(x, \pm y)=p \circ \phi^{ \pm}(x, y),
$$

the matrices $K^{ \pm}=\left(\nabla^{T}\left(\phi^{ \pm}\right)^{-1}\right)^{T}=\left(\begin{array}{ll}1 & \pm 1 \\ 0 & \mp 1\end{array}\right)$, the differential operator $D_{\lambda}=$ $\left(\partial_{x}, \lambda\right)$, and the operators

$$
A^{ \pm}\left(x, y, \xi, \partial_{x}\right)=A\left(x, y, K^{ \pm} D_{-\theta^{ \pm}(\xi)}^{T}\right) \text { and } B^{ \pm}\left(x, y, \xi, \partial_{x}\right)=B\left(x, y, K^{ \pm} D_{-\theta^{ \pm}(\xi)}^{T}\right) .
$$

3.1. Equations of complex symbols. Here we prove that if the linear operator with kernel $p$ admits a $\theta^{ \pm}$-diffusive realization, then its symbols $\mu^{ \pm}$are a solution to the equations

$$
\begin{aligned}
& \pm\left\langle A^{ \pm} \mu^{ \pm}, e^{\mp \theta(\xi)(x-y)}\right\rangle=q \text { in } \Omega^{ \pm}, \\
& \pm\left\langle B^{ \pm} \mu^{ \pm}, e^{\mp \theta(\xi)(x-y)}\right\rangle=r \text { on } \partial \Omega^{ \pm} .
\end{aligned}
$$

Then, where $A$ and $B$ are independent of $y$, we replace equation (24) and equation (25) restricted to $\Gamma_{y}^{ \pm}$by two infinite families of differential equations in $x$ parameterized by $\xi$.

The Laplace transforms of the extension $y \mapsto \widetilde{p}^{ \pm}(., y)$ being denoted by $\lambda \mapsto$ $\mathcal{P}^{ \pm}(., \lambda)$, as in (16), we make assumptions allowing calculations as in the definition of complex symbols: 
Assumption 14. The functions $\lambda \mapsto \mathcal{P}^{ \pm}(x, \lambda), \lambda \mapsto A\left(x, y, K^{ \pm} D_{\lambda}^{T}\right) \mathcal{P}^{ \pm}(x, \lambda)$ for $(x, y) \in \omega \times \mathbb{R}$ and $\lambda \mapsto B\left(x, y, K^{ \pm} D_{\lambda}^{T}\right) \mathcal{P}^{ \pm}(x, \lambda)$ for $(x, y) \in \partial \Omega^{ \pm}$satisfy Assumption 4.

Proposition 15. Under Assumptions 14, $p$ is a solution to the boundary value problem (21) -(22) if and only if its symbols $\mu^{ \pm}$are solution to the system (24)(25).

Proof. Changing the variables in (21)-(22), the equations satisfied by $\widetilde{p}^{ \pm}$are

$$
A\left(\phi^{ \pm}(x, y), K^{ \pm} \nabla\right) \widetilde{p}^{ \pm}=q \circ \phi^{ \pm} \text {and } B\left(\phi^{ \pm}(x, y), K^{ \pm} \nabla\right) \widetilde{p}^{ \pm}=r \circ \phi^{ \pm} .
$$

We introduce the Laplace transforms $\mathcal{P}^{ \pm}$of $\widetilde{p}^{ \pm}$, so $\widetilde{p}^{ \pm}=\mathcal{L}^{-1} \mathcal{L} \widetilde{p}^{ \pm}=\mathcal{L}^{-1} \mathcal{P}^{ \pm}$and

$$
\begin{aligned}
\frac{1}{2 i \pi} \int_{a+i \mathbb{R}} A\left(\phi^{ \pm}(x, y), K^{ \pm} D_{\lambda}^{T}\right) \mathcal{P}^{ \pm}(x, \lambda) e^{\lambda y} d \lambda & =q \circ \phi^{ \pm}, \\
\frac{1}{2 i \pi} \int_{a+i \mathbb{R}} B\left(\phi^{ \pm}(x, y), K^{ \pm} D_{\lambda}^{T}\right) \mathcal{P}^{ \pm}(x, \lambda) e^{\lambda y} d \lambda & =r \circ \phi^{ \pm},
\end{aligned}
$$

for a convenient $a \in \mathbb{R}$. Changing the integration path as in the proof of Theorem 8 yields

$$
\begin{aligned}
& \pm\left\langle A\left(\phi^{ \pm}(x, y), K^{ \pm} D_{-\theta^{ \pm}(\xi)}^{T}\right) \mu^{ \pm}(x, \xi), e^{-\theta^{ \pm}(\xi) y}\right\rangle_{\xi}=q \circ \phi^{ \pm}, \\
& \pm\left\langle B\left(\phi^{ \pm}(x, y), K^{ \pm} D_{-\theta^{ \pm}(\xi)}^{T}\right) \mu^{ \pm}(x, \xi), e^{-\theta^{ \pm}(\xi) y}\right\rangle_{\xi}=r \circ \phi^{ \pm},
\end{aligned}
$$

provided that $\lambda \mapsto \mathcal{P}^{ \pm}(x, \lambda), \lambda \mapsto A\left(\phi^{ \pm}(x, y), K^{ \pm}\left(\partial_{x}, \lambda\right)^{T}\right) \mathcal{P}^{ \pm}(x, \lambda)$ and $\lambda \mapsto$ $B\left(\phi^{ \pm}(x, y), K^{ \pm}\left(\partial_{x}, \lambda\right)^{T}\right) \mathcal{P}^{ \pm}(x, \lambda)$ are holomorphic in $D^{ \pm}$and decrease at infinity. Inverting the change of variable leads to the result,

$$
\begin{aligned}
& \pm\left\langle A\left(x, y, K^{ \pm} D_{-\theta^{ \pm}(\xi)}^{T}\right) \mu^{ \pm}(x, \xi), e^{\mp \theta(\xi)(x-y)}\right\rangle_{\xi}=q, \\
& \pm\left\langle B\left(x, y, K^{ \pm} D_{-\theta^{ \pm}(\xi)}^{T}\right) \mu^{ \pm}(x, \xi), e^{\mp \theta(\xi)(x-y)}\right\rangle_{\xi}=r .
\end{aligned}
$$

Now, for an operator $A$ and an operator $B$ restricted to $\Gamma_{y}^{ \pm}$, both being independent of $y$, we prove that the related symbol equations can be rewritten in the Laplace domain. We therefore consider the corresponding right-hand side $q$ and $r$ as the kernels of certain operators. We assume that their impulse responses $y \mapsto \widetilde{q}$ and $y \mapsto \widetilde{r}$ satisfy Assumptions 4 for the same set $D^{ \pm}$as $p$. Thus, they admit $\theta^{ \pm}$-diffusive realizations with symbols denoted by $\nu^{ \pm}$and $\rho^{ \pm}$. If we apply the same treatment to $q$ and $r$ as to the left-hand side, we obtain

$$
\begin{aligned}
& \left\langle A\left(x, K^{ \pm} D_{-\theta^{ \pm}}^{T}\right) \mu^{ \pm}-\nu^{ \pm}, e^{\mp \theta(x-y)}\right\rangle=0, \\
& \left\langle B\left(x, K^{ \pm} D_{-\theta^{ \pm}}^{T}\right) \mu^{ \pm}-\rho^{ \pm}, e^{\mp \theta(x-y)}\right\rangle=0 .
\end{aligned}
$$

Referring to Remark 10 about the lack of uniqueness of a diffusive realization, we could not eliminate the brackets to obtain local equations on the complex diffusive symbols. So, we proceed in a different way.

We start by extending the equations of $\widetilde{p}^{ \pm}$to $\omega \times \mathbb{R}^{+}$. According to Remark 9 , the functions $y \mapsto \widetilde{q}^{ \pm}$and $y \mapsto \widetilde{r}^{ \pm}$are necessarily analytic, and their extensions over $\mathbb{R}^{+}$used for Laplace transforms are necessarily the analytic extensions. After extending the functions, the equations (with constant coefficients in the $y$-direction) are straightforwardly extended. Then, $y \mapsto \widetilde{p}^{ \pm}, y \mapsto \widetilde{q}^{ \pm}$and $y \mapsto \widetilde{r}^{ \pm}$for $y \in \Gamma_{y}^{ \pm}$ 
are extended by 0 to $\mathbb{R}^{-}$, and we know that there exist suitable partial differential operators $A_{k}^{ \pm}(x, \nabla)$ and $B_{k}^{ \pm}(x, \nabla)$ such that the following equations, written in the distribution sense in $D_{+}^{\prime}$ with respect to $y$,

$$
\begin{aligned}
A\left(x, K^{ \pm} \nabla\right) \widetilde{p}^{ \pm}+\sum_{k} A_{k}^{ \pm}\left(x, K^{ \pm} \nabla\right) \widetilde{p}^{ \pm} \delta_{0}^{(k)} & =\widetilde{q}^{ \pm}, \\
\text {and } B\left(x, K^{ \pm} \nabla\right) \widetilde{p}^{ \pm}+\sum_{k} B_{k}^{ \pm}\left(x, K^{ \pm} \nabla\right) \widetilde{p}^{ \pm} \delta_{0}^{(k)} & =\widetilde{r}^{ \pm},
\end{aligned}
$$

are equivalent to equation (21) and boundary conditions (22) on $\Gamma_{y}^{ \pm}$. Here $\delta_{0}^{(k)}$ is the $k^{t h}$ derivative of the Dirac distribution at 0 . From $A_{k}^{ \pm}$and $B_{k}^{ \pm}$we define the integro-differential operators $A_{0}^{ \pm}$and $B_{0}^{ \pm}$as

$$
\begin{aligned}
& A_{0}^{ \pm}\left(x, \partial_{x}, \xi\right) \mu(x, \xi)=\mp \frac{\theta^{ \pm \prime}(\xi)}{2 i \pi} \sum_{k}\left(-\theta^{ \pm}(\xi)\right)^{k}\left\langle A_{k}^{+}\left(x, K^{ \pm} D_{-\theta^{ \pm}(\zeta)}^{T}\right) \mu(x, \zeta), 1\right\rangle_{\zeta}, \\
& B_{0}^{ \pm}\left(x, \partial_{x}, \xi\right) \mu(x, \xi)=\mp \frac{\theta^{ \pm \prime}(\xi)}{2 i \pi} \sum_{k}\left(-\theta^{ \pm}(\xi)\right)^{k}\left\langle B_{k}^{+}\left(x, K^{ \pm} D_{-\theta^{ \pm}(\zeta)}^{T}\right) \mu(x, \zeta), 1\right\rangle_{\zeta} .
\end{aligned}
$$

Proposition 16. The operator $A$ and the operator $B$ restricted to $\Gamma_{y}^{ \pm}$being independent of $y$, the kernel $p(x, y)$ is a solution to (24) with boundary conditions (25) in $\Gamma_{y}^{ \pm}$iff the symbols $\mu^{ \pm}$are a solution to

$$
\begin{gathered}
\left(A^{ \pm}+A_{0}^{ \pm}\right) \mu^{ \pm}=\nu^{ \pm} \text {in } \omega \times \mathbb{R}, \\
\left(B^{+}+B_{0}^{+}\right) \mu^{+}=\rho^{+} \text {in }\{1\} \times \mathbb{R} \text { and }\left(B^{-}+B_{0}^{-}\right) \mu^{-}=\rho^{-} \text {in }\{0\} \times \mathbb{R} .
\end{gathered}
$$

Proof. We apply the Laplace transform to equations (27)-(28) in the sense of distributions

$$
\begin{aligned}
& A\left(x, K^{ \pm} D_{\lambda}\right) \mathcal{P}^{ \pm}+\sum_{k} \lambda^{k} A_{k}^{ \pm}\left(x, K^{ \pm} \nabla\right) \widetilde{p}^{ \pm}(x, 0)=\mathcal{L}\left(\widetilde{q}^{ \pm}\right), \\
& B\left(x, K^{ \pm} D_{\lambda}\right) \mathcal{P}^{ \pm}+\sum_{k} \lambda^{k} B_{k}^{ \pm}\left(x, K^{ \pm} \nabla\right) \widetilde{p}^{ \pm}(x, 0)=\mathcal{L}\left(\widetilde{r}^{ \pm}\right) .
\end{aligned}
$$

Since the Laplace transforms of $\widetilde{p}^{ \pm}, \widetilde{q}^{ \pm}$and of $\widetilde{r}^{ \pm}$are all holomorphic in $D^{ \pm}$these equations are still true along the parameterized paths $\xi \mapsto \theta^{ \pm}(\xi)$. Changing the variable $\lambda$ into $\xi$ and introducing the diffusive symbols yields

$$
\begin{aligned}
& A^{ \pm} \mu^{ \pm} \mp \frac{\theta^{ \pm}}{2 i \pi} \sum_{k}\left(-\theta^{ \pm}\right)^{k} A_{k}^{ \pm}\left(x, K^{ \pm} \nabla\right) \widetilde{p}^{ \pm}(x, 0)=\nu^{ \pm}, \\
& B^{ \pm} \mu^{ \pm} \mp \frac{\theta^{ \pm}}{2 i \pi} \sum_{k}\left(-\theta^{ \pm}\right)^{k} B_{k}^{ \pm}\left(x, K^{ \pm} \nabla\right) \widetilde{p}^{ \pm}(x, 0)=\rho^{ \pm} .
\end{aligned}
$$

To conclude the proof, we introduce $\widetilde{p}^{ \pm}=\mathcal{L}^{-1} \mathcal{L}(p)$ and proceed as in the proof of Proposition 15] to get

$$
A_{k}^{ \pm}\left(x, K^{ \pm} \nabla\right) \widetilde{p}^{ \pm}(x, 0)=\left\langle A_{k}^{ \pm}\left(x, K^{ \pm} D_{-\theta^{ \pm}(\zeta)}^{T}\right) \mu^{ \pm}(., \zeta), 1\right\rangle_{\zeta} .
$$

Remark 17. To close this section we discuss the a priori determination of $D^{ \pm}$. A singularity occurs in a solution to the equations in a Laplace domain when an operator coefficient vanishes or when a right-hand side is singular. The second possibility is already taken into account explicitly in our previous assumptions. We 
consider situations where the mappings $\lambda \mapsto A\left(x, \partial_{x}, \lambda\right)$ and $\lambda \mapsto B\left(x, \partial_{x}, \lambda\right)$ are analytic and where the operators $A$ and $B$ can be expanded as finite series in $\partial_{x}$,

$$
A\left(x, K^{ \pm} D_{\lambda}^{T}\right)=\sum_{m} a_{m}^{ \pm}(x, \lambda) \partial_{x}^{m} \text { and } B\left(x, K^{ \pm} D_{\lambda}^{T}\right)=\sum_{m} b_{m}^{ \pm}(x, \lambda) \partial_{x}^{m} .
$$

Then, $\lambda \mapsto P^{ \pm}(., \lambda)$ is potentially singular at the zeros of the mappings $\lambda \mapsto$ $a_{m}^{ \pm}(x, \lambda)$ and $\lambda \mapsto b_{m}^{ \pm}(x, \lambda)$. In the forthcoming applications, we refer to these sets as $W_{A}^{ \pm}$and $W_{B}^{ \pm}$, namely

$$
W_{A}^{ \pm}:=\bigcup_{x, m}\left[a_{m}^{ \pm}(x, .)\right]^{-1}(0) \text { and } W_{B}^{ \pm}:=\bigcup_{x, m}\left[b_{m}^{ \pm}(x, .)\right]^{-1}(0) .
$$

3.2. Equations of real symbols. Now, we establish the symbol equations in the framework of real diffusive symbols introduced in Section 2.3. We choose $\theta^{ \pm *} \subset \mathbb{R}$ as in (19) with $\sigma^{ \pm}=1$, so

$$
\theta^{ \pm *}(\xi)=\lambda_{0}^{ \pm}+\xi \text { where } \xi \in \mathbb{R}^{+} .
$$

The real symbols of $q$ and $r$ are denoted by $\nu^{ \pm *}$ and $\rho^{ \pm *}$.

Proposition 18. (i) Assuming that $p, q=A(x, y, \nabla) p$ and $r=B(x, y, \nabla) p$ satisfy Assumption 12, then $p$ is a solution to the boundary value problem (21)-(22) iff its real diffusive symbols $\mu^{ \pm *}$ are solution to the system

$$
\begin{aligned}
& \pm\left\langle A^{ \pm} \mu^{ \pm *}, e^{\mp \theta^{ \pm *}(\xi)(x-y)}\right\rangle_{\xi}=q \text { in } \Omega^{ \pm}, \\
& \pm\left\langle B^{ \pm} \mu^{ \pm *}, e^{\mp \theta^{ \pm *}(\xi)(x-y)}\right\rangle_{\xi}=r \text { on } \partial \Omega^{ \pm} .
\end{aligned}
$$

(ii) Moreover, if the operator $A$ and the operator $B$ restricted to $\Gamma_{y}^{ \pm}$are independent of $y$, then $p$ is a solution to equation (21) and to boundary condition (22) on $\Gamma_{y}^{ \pm}$iff $\mu^{ \pm *}$ are the solution to

$$
\begin{gathered}
A^{ \pm} \mu^{ \pm *}=\nu^{ \pm *} \text { in } \omega \times \mathbb{R}^{+}, \\
B^{+} \mu^{+*}=\rho^{+*} \text { in }\{1\} \times \mathbb{R}^{+} \text {and } B^{-} \mu^{-*}=\rho^{-*} \text { in }\{0\} \times \mathbb{R}^{+} .
\end{gathered}
$$

Proof. (i) We already know that $\widetilde{p}^{ \pm}$is the solution to (26), and we use its expression (20) to get

$$
\begin{aligned}
& \pm\left\langle A\left(\phi^{ \pm}(x, y), K^{ \pm} D_{-\theta^{ \pm *}}^{T}\right) \mu^{ \pm *}, e^{-\theta^{ \pm *} y}\right\rangle=q \circ \phi^{ \pm}, \\
& \pm\left\langle B\left(\phi^{ \pm}(x, y), K^{ \pm} D_{-\theta^{ \pm}}^{T}\right) \mu^{ \pm *}, e^{-\theta^{ \pm *} y}\right\rangle=r \circ \phi^{ \pm} .
\end{aligned}
$$

The announced equations follow after changing the variables back to the original ones.

(ii) Introducing the diffusive representations of $q$ and $r$ in (36), (37) yields

$$
\left\langle A^{ \pm} \mu^{ \pm *}, e^{-\theta^{ \pm *} y}\right\rangle=\left\langle\nu^{ \pm *}, e^{-\theta^{ \pm *} y}\right\rangle \text { and }\left\langle B \mu^{ \pm *}, e^{-\theta^{ \pm *} y}\right\rangle=\left\langle\rho^{ \pm *}, e^{-\theta^{ \pm *} y}\right\rangle,
$$

or equivalently, when replacing $\theta^{ \pm *}$ by $\lambda_{0}^{ \pm *}+\xi$ :

$$
\mathcal{L}\left(A^{ \pm} \mu^{ \pm *}\right)=\mathcal{L}\left(\nu^{ \pm *}\right) \text { and } \mathcal{L}\left(B \mu^{ \pm *}\right)=\mathcal{L}\left(\rho^{ \pm *}\right) \text { for Rey } \geq 0 .
$$

Since the Laplace transform is injective in $\mathcal{D}_{+}^{\prime}$, we get the equalities (34), (35). 


\section{An illustrative example}

The Lyapunov equation under consideration appears in the context of internal stabilization of the heat equation with Dirichlet boundary conditions. We consider a nonnegative constant $c$ and a positive, self-adjoint operator $Q \in \mathcal{L}\left(L^{2}(\omega)\right)$ with kernel $q$, and admitting diffusive representations with symbols $\nu^{ \pm}$. The Lyapunov equation states: Find $P \in L\left(H_{0}^{1}(\omega)\right)$ such that

$$
\int_{\omega} \frac{d u}{d x} \frac{d(P v)}{d x}+\frac{d\left(P^{*} u\right)}{d x} \frac{d v}{d x} d x=\int_{\omega} Q u v+c u v d x \text { for all } u, v \in H_{0}^{1}(\omega) .
$$

We will seek the solution $P$ as a kernel operator with kernel $p(x, y)$, the kernel of its adjoint $P^{*}$ being $p^{*}(x, y)=p(y, x)$. We can readily prove that $p$ is symmetric, i.e., that $p^{*}=p$, by interchanging $u$ with $v$, and then $x$ with $y$. Now, we derive the equations satisfied by the kernel $p$ in $\Omega^{+}$and in $\Omega^{-}$in the form (21), (22). For brevity, we use the notations $p^{+}$and $p^{-}$for $p_{\mid \Omega^{+}}$and $p_{\mid \Omega^{-}}$the kernel causal and anti-causal parts.

Proposition 19. The causal part $p^{+}$and the anti-causal part $p^{-}$of the kernel $p$ are the unique solutions to the two uncoupled boundary value problems

$$
\begin{aligned}
-\Delta p^{ \pm} & =q^{ \pm} \text {in } \Omega^{ \pm}, \\
\left(-\partial_{x}+\partial_{y}\right) p^{ \pm} & = \pm \frac{c}{2} \text { on } \Gamma_{0}, p^{ \pm}=0 \text { on } \Gamma_{x}^{ \pm} \cup \Gamma_{y}^{ \pm} .
\end{aligned}
$$

In the particular case $c=0, p$ is the unique solution to

$$
-\Delta p=q \text { in } \Omega \text { and } p=0 \text { on } \partial \Omega \text {. }
$$

Proof. Using the integral forms of $P u$, of $P v$ and of $Q u$ in the Lyapunov equation leads to

$$
\begin{gathered}
\int_{\Omega} \partial_{x} u(x) \partial_{x}(p(x, y) v(y))+\partial_{x}(p(x, y) u(y)) \partial_{x} v(x) d y d x \\
=\int_{\omega} c u(x) v(x) d x+\int_{\Omega} q(x, y) u(x) v(y) d y d x
\end{gathered}
$$

We introduce $w(x, y)=u(x) v(y) \in H_{0}^{1}(\Omega)$ and we use the symmetry $p(x, y)=$ $p(y, x)$ to obtain the variational formulation satisfied by $p$,

$$
\int_{\Omega} \nabla p \nabla w d y d x=\frac{1}{\sqrt{2}} \int_{\Gamma_{0}} c w d s+\int_{\Omega} q w d y d x .
$$

Furthermore, we remark that the set of functions $w(x, y)=u(x) v(y)$, with $u$, $v \in H_{0}^{1}(\omega)$, is dense in $H_{0}^{1}(\Omega)$ and that $p=0$ on $\partial \Omega$ because $P u=0$ on $\partial \omega$ and because $p$ is symmetric. So $p \in H_{0}^{1}(\Omega)$ is the solution to (41) for any $w \in H_{0}^{1}(\Omega)$. This variational formulation fulfills the Lax-Milgram Lemma assumptions, and therefore admits a unique solution. When $c$ vanishes, the variational formulation is straightforwardly interpreted as (40). When $c \neq 0$, the strong form of the equation must be written on each side of $\Gamma_{0}$. Applying the Green formula in $\Omega-\Gamma_{0}=\Omega^{+} \cup \Omega^{-}$ we find

$$
\int_{\Omega^{+} \cup \Omega^{-}}-(\Delta p+q) w d y d x+\int_{\Gamma_{0}} \frac{1}{\sqrt{2}}\left(-\partial_{x}+\partial_{y}\right)\left(p^{+}-p^{-}-c\right) w d s=0,
$$


which yields the strong formulation

$$
\begin{aligned}
-\Delta p & =q \text { on } \Omega^{+} \cup \Omega^{-}, \\
\left(-\partial_{x}+\partial_{y}\right)\left(p^{+}-p^{-}\right) & =c \text { on } \Gamma_{0} \text { and } p=0 \text { on } \partial \Omega .
\end{aligned}
$$

Since $p$ is symmetric, the second relation is equivalent to one of the two conditions,

$$
\left(-\partial_{x}+\partial_{y}\right) p^{+}=\frac{c}{2} \quad \text { or } \quad\left(-\partial_{x}+\partial_{y}\right) p^{-}=-\frac{c}{2},
$$

which ends the proof.

4.1. Complex diffusive realizations. Here, we apply the general theory introduced for complex symbol equations. We start by writing equations (24) and (25) of the symbols corresponding to the boundary value problem (39). We find that $A^{ \pm} \mu^{ \pm}=\left(\partial_{x x}^{2} \mp 2 \theta^{ \pm} \partial_{x}+2 \theta^{ \pm 2}\right) \mu^{ \pm}$with $B^{ \pm} \mu^{ \pm}=\mu^{ \pm}$for Dirichlet conditions and with $B^{ \pm} \mu^{ \pm}=\left(\partial_{x} \mp 2 \theta^{ \pm}\right) \mu^{+}$for the Neuman condition on $\Gamma_{0}$. We further assume that $q$ is such that Assumption 14 is satisfied. Thus, applying Proposition 15yields the set of equations (24) and (25) which, in this case, are rewritten as

$$
\begin{gathered}
\pm\left\langle\left(\partial_{x x}^{2}-2 \theta^{ \pm} \partial_{x}+2 \theta^{ \pm 2}\right) \mu^{ \pm}, e^{\mp \theta^{ \pm}(x-y)}\right\rangle=q(x, y) \text { in } \Omega^{ \pm}, \\
\left\langle\left(\partial_{x} \mp 2 \theta^{ \pm}\right) \mu^{ \pm}, 1\right\rangle=0 \text { in } \omega, \\
\left\langle\mu^{+}, e^{-\theta^{+} x}\right\rangle=0,\left\langle\mu^{-}, e^{\theta^{-}(x-1)}\right\rangle=0 \text { in } \omega, \\
\left\langle\mu^{+}, e^{-\theta^{+}(1-y)}\right\rangle=0 \text { on } \Gamma_{y}^{+} \text {, and }\left\langle\mu^{-}, e^{-\theta^{-} y}\right\rangle=0 \text { on } \Gamma_{y}^{-} .
\end{gathered}
$$

According to Remark 17, the sets of possible singularities related to the interior equations and to the boundary conditions are $W_{A}^{ \pm}=\{0\}$ and $W_{B}^{ \pm}=\emptyset$.

4.2. Real diffusive realizations. In this subsection, we introduce a formal calculation technique for real diffusive symbols. We think that it could also be used as a starting point for a numerical method. We limit our presentation to the case $q=0$ but $c \neq 0$. Since the set of possible singularities $W_{A}^{ \pm} \cup W_{B}^{ \pm}=\{0\}$ in the diffusive symbols is included in $\mathbb{R}^{-}$, we can apply the framework of real diffusive symbols. We choose the paths $-\theta^{ \pm *}=\mathbb{R}^{-} \supset W_{A}^{ \pm} \cup W_{B}^{ \pm}$(i.e., $\lambda_{0}^{ \pm}=0$ and $\sigma^{ \pm}=1$ ) and we apply Proposition 18. The diffusive symbol equations turn out to be

$$
\begin{gathered}
\left(\partial_{x x}^{2}-2 \theta^{ \pm *} \partial_{x}+2 \theta^{ \pm * 2}\right) \mu^{ \pm *}=0 \text { in } \omega \times \mathbb{R}^{+}, \\
\left\langle\left(\partial_{x} \mp 2 \theta^{ \pm *}\right) \mu^{ \pm *}, 1\right\rangle=\mp \frac{c}{2} \text { in } \omega, \\
\left\langle\mu^{+*}, e^{-\theta^{+*} x}\right\rangle=0,\left\langle\mu^{-*}, e^{\theta^{-*}(x-1)}\right\rangle=0 \text { in } \omega, \\
\mu^{+*}(1, .)=0 \text { in } \mathbb{R}^{+}, \text {and } \mu^{-*}(0, .)=0 \text { in } \mathbb{R}^{+} .
\end{gathered}
$$

As mentioned before, in such a case diffusive symbols are not functions, and a suitable duality has to be chosen. We do not develop a complete theory for the equations of $\mu^{ \pm *}$ but we give their solutions and we propose a formal general method for their derivation. The formal method consists in seeking a solution $\mu^{ \pm *}$ as a sum of a regular part $g^{ \pm}$and of a series of Dirac mass derivatives concentrated at the potential singularities. Thus we seek a solution for the causal part as

$$
\mu^{+*}(x, \xi)=g(x, \xi)+\sum_{n=0}^{n_{0}} g_{n}(x) \delta^{(n)}(\xi)
$$


where $n_{0}$ is assumed to be an a priori unknown finite positive integer, and we express $\mu^{-*}$ with respect to $\mu^{+*}$.

Proposition 20. If $Q=0$ and $\theta^{ \pm *}(\xi)=\xi \in \mathbb{R}^{+}$, then $P$ admits a $\theta^{ \pm *}$-diffusive realization in the form (50) with $g=0$ and $n_{0}=1$,

$$
\mu^{+*}(x, \xi)=\frac{c}{2}(1-x)\left(x \delta(\xi)+\delta^{\prime}(\xi)\right) \text { in } \omega \times \mathbb{R}^{+} .
$$

Moreover,

$$
\mu^{-*}(x, \xi)=\mu^{+*}(1-x, \xi) \text { in } \omega \times \mathbb{R}^{+} .
$$

Proof. (i) First, we state the relation between $\mu^{+*}$ and $\mu^{-*}$. Here, $P$ preserves the symmetry for the center of the interval $\omega$, in the sense that $P u(x)=P u(1-x)$ for any $u$ such that $u(y)=u(1-y)$. Then,

$p(x, y)=p(1-x, 1-y), \widetilde{p}(x, y)=\widetilde{p}(x-y,-y)$ and $\mu^{-*}(1-x, \xi)=\mu^{+*}(x, \xi) \forall x \in \omega$.

(ii) We proceed by trying, successively, solutions

$$
\mu^{+*}=g, \mu^{+*}=g+g_{0} \delta, \mu^{+*}=g+g_{0} \delta+g_{1} \delta^{\prime}, \ldots .
$$

The first two cases do not yield a solution, but by inserting the third case in the set of equations we find $g=0, g_{0}(x)=c x(1-x) / 2$ and $g_{1}(x)=c(x-1) / 2$. Since we know that the solution exists and is unique, the conclusion follows. Notice that our calculation requires the results stated in Lemma 23 from the Appendix established for a test function in $\varphi \in D(R)$ and applied to $\varphi \equiv 1$ which allows the same calculation for distributions with support in a bounded set. Note that we have used the results summarized in the Appendix for a sequence of test functions $\varphi_{n}$ converging to 1 when $n$ tends to infinity.

\section{Numerical APPROXIMATIONS AND RELAXATION OF ASSUMPTIONS}

The approximations presented in this section are formulated in the particular case of Lyapunov equation (38). In Subsection 5.1. we present our approximation of a diffusive realization derived from an approximation of the kernels $p^{ \pm}$. In Subsection 5.2. we introduce two weak formulations posed directly on the diffusive symbols. We recall that all these discretization methods are kept at a distance from the continuous case in the sense that they do not provide converging approximations of the analytic extensions of impulse responses nor converging approximations of diffusive symbols.

5.1. Diffusive symbol approximation from kernel approximation. Here we report results obtained for approximation of a diffusive realization based on a dedicated spectral approximation of the kernel $p$. For the sake of simplicity, we have restricted our calculations to the situation where $c=0$ in the Lyapunov equation, so we consider the solution of the weak formulation associated with (40). The spectral method uses a polynomial basis in $x$ together with a positive exponential basis in $y$ for the causal part and a negative exponential basis in $y$ for the anti-causal part. In both cases, the calculations have been carried out in the entire set $\Omega$. Our approximations are of the form

$$
p^{N \pm}(x, y)=\sum_{k=0}^{N_{1}} \sum_{\ell=0}^{N_{2}}(1-x) x^{k+1}\left(1-e^{ \pm y}\right)\left(e^{ \pm 1}-e^{ \pm y}\right) e^{ \pm \ell y} p_{k \ell}^{ \pm},
$$


designed to satisfy the Dirichlet boundary conditions. Note that $D^{N \pm}$, the domains of holomorphy can be any open set not containing the negative real numbers $\left\{0,-1, \ldots,-N_{2}\right\}$. Assumption 4 is clearly satisfied for any contours $-\theta^{ \pm}$in $D^{N \pm}$, and we may apply Theorem 8 to derive the corresponding complex symbols $\mu^{N \pm}$. The approximate diffusive realizations is then $u \mapsto\left\langle\mu^{N \pm}, \psi^{ \pm}(u)\right\rangle$. To calculate $\mu^{N \pm}$, we have chosen the contours $-\theta^{ \pm}$proposed by J.A.C. Weideman and L.N. Trefethen in [32, in the context of inverse Laplace transform computation, namely a parabola,

$$
-\theta^{ \pm}(\xi)=\theta_{p}(i \xi+1)^{2} \text { for } \xi \in \mathbb{R}
$$

and a hyperbola,

$$
-\theta^{ \pm}(\xi)=\theta_{h}(1+\sin (i \xi-\alpha)) \text { for } \xi \in \mathbb{R},
$$

for some positive real numbers $\theta_{p}, \theta_{h}$ and $\alpha$ the hyperbola asymptotic angle.

Discretization of $\psi$ with respect to $x$. Two $x$-discretizations have been considered. They are based on two interpolations of the discrete inputs $\left(u_{n}\right)_{n \in\{0, \ldots, \mathcal{N}\}}$, the first one being piecewise constant, and the second one globally continuous and piecewise linear on each interval $\left[x_{n}, x_{n+1}\right]$. The discretization step is kept constant $h=x_{n+1}-x_{n}$. In the linear interpolation case, we obtained the recurrence relation on $\psi_{n}^{ \pm}$from the integral forms (11) of $\psi^{ \pm}$,

$$
\begin{aligned}
\psi_{n+1, k}^{+} & =\alpha_{k}^{+} \psi_{n, k}^{+}+\beta_{k}^{+} u_{n}+\gamma_{k}^{+} u_{n+1}, & & \psi_{0, k}^{+}=0, \\
\psi_{n, k}^{-} & =\alpha_{k}^{-} \psi_{n+1, k}^{-}+\beta_{k}^{-} u_{n}+\gamma_{k}^{-} u_{n+1}, & & \psi_{\mathcal{N}, k}^{-}=0 .
\end{aligned}
$$

In the piecewise constant interpolation case, the formulas for $\psi_{n}^{ \pm}$have the same forms without terms $\gamma_{k}^{ \pm} u_{n+1}$.

Finally, the integrals along $-\theta^{ \pm}$are approximated by an ordinary trapezoidal quadrature rule using $M$ points.

Remark 21. The estimation error of numerical integrations along the paths $-\theta^{ \pm}$ are linked to the numerical inversion of the Laplace transform. We use the related error estimates established by Trefethen et al. in 32 for parabolic and hyperbolic paths expressed for constant and linear approximations of $u$. This yields optimal relations between the number $M$ of quadrature nodes and the length $h$ in each case, so that the errors turn out to be regulated by the single parameter $h$ only. More details will be reported in a paper on numerical issues.

In our presentation of numerical experiments, we discuss only causal parts. Similar results have been observed for anti-causal parts. We have considered the kernel $q(x, y)=2(1-3 x)(1-y) y^{2}+2(1-x) x^{2}(1-3 y)$ and the input variable $u(x)=\sin (\pi x)$. The polynomial degrees of $p^{N+}$ are fixed at sufficiently large values, $N_{1}=N_{2}=15$, so that the relative error

$$
e_{N}^{+}=\frac{\left\|p^{+}-p^{N+}\right\|_{L^{2}(\Omega)}}{\left\|p^{+}\right\|_{L^{2}((\Omega))}}
$$

is negligible, namely in the order of magnitude of $10^{-9}$. In Figure 2 we report the relative errors in logarithmic scale between $P^{+} u$ an exact realization, and various approximations $P^{h+} u$ parameterized by $h$ only,

$$
E^{h+}=\frac{\left\|P^{+} u-P^{h+} u\right\|_{L_{h}^{2}(\omega)}}{\left\|P^{+} u\right\|_{L_{h}^{2}(\omega)}} .
$$




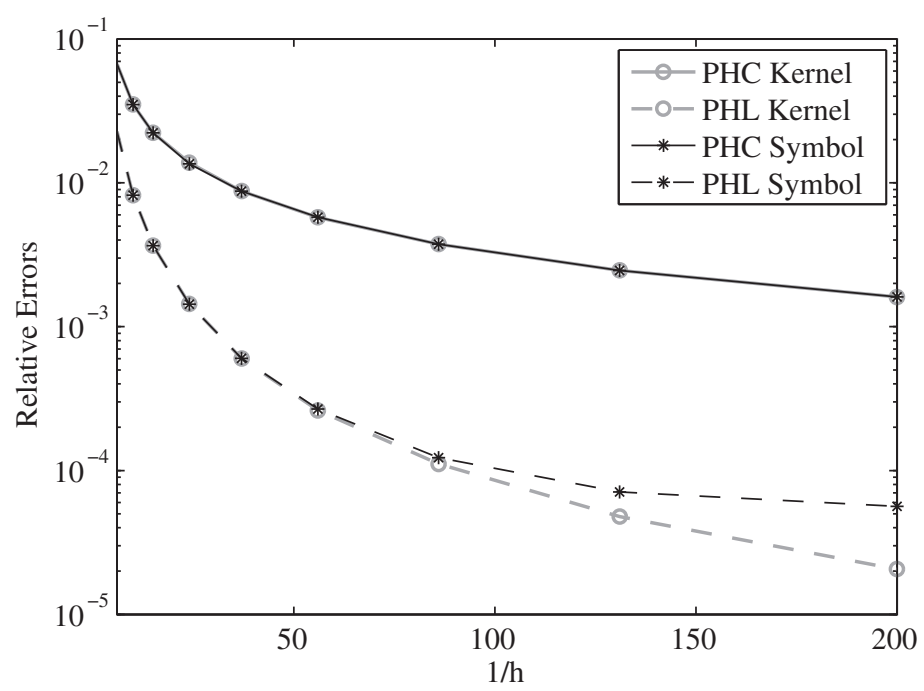

Figure 2. Errors between $P^{+} u$ and $P^{h+} u$

The error is measured by the discrete $L^{2}(\omega)$-norm, and the discretization step $h$ ranges from 0.005 to 0.25 . For the approximation method of $\mu^{+}$via the kernel (Kernel), the errors decay rate is proportional to $h$ for piecewise constant (C) interpolation, and proportional to $h^{2}$ for piecewise linear $(\mathrm{L})$ interpolation. Note that our diffusive realization approximation has two other error sources. The first originates from spectral approximation of $\mu$, and it is negligible in our calculation. The second comes from discretization of contour integrals which is linked to $h$, as explained in Remark 21. This is why the results related to parabolic (P) and hyperbolic $(\mathrm{H})$ are represented on the same curves.

Computation time. Even if our goal in developing diffusive realizations $u \mapsto P u$ of operators is oriented towards implementation in semi-decentralized computer architectures, it is worthwhile comparing our approach to a classical one, namely a direct (Di) quadrature method,

$$
P u\left(x_{n}\right) \approx h \sum_{j=0}^{\mathcal{N}} p\left(x_{n}, y_{j}\right) u\left(y_{j}\right), \text { for } n=0, . ., \mathcal{N} .
$$

Figure 3 provides a comparison of computation times between the direct quadrature method, and the diffusive realization methods with hyperbolic $(\mathrm{H})$ and parabolic (P) contours, both with piecewise constant interpolation of $u$. We notice that hyperbolic paths always yield faster computation over parabolic ones. The gain in using diffusive realization over direct quadrature increases for finer spatial discretization points. We underline that we are aware that our simulations have been conducted for an elementary input $u$ and that further tests are needed to yield a more general conclusion.

Remark 22. We have used a spectral method to discretize both $x$ - and $y$-directions. In the $y$-direction we actually need to use global basis functions so that they can be analytically extended. On the contrary, there is no particular restriction regarding 


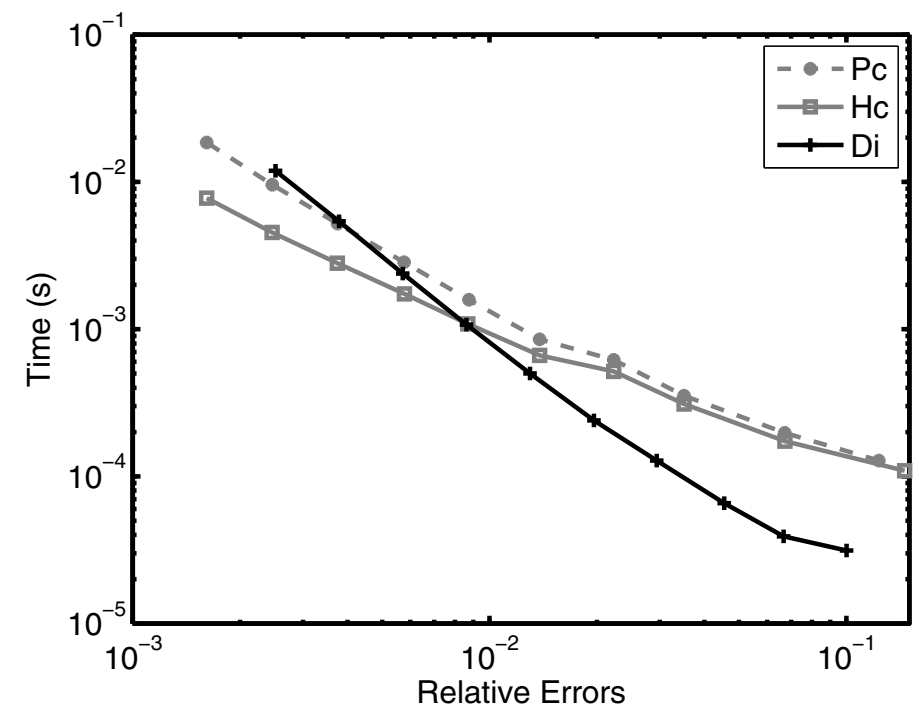

Figure 3. Computation time in seconds versus relative error $E^{h+}$

approximations in the $x$-direction. For instance, a local basis as a finite element basis might be used.

5.2. Diffusive symbol approximation from symbol approximation. Here, we introduce two pairs of weak formulations for the symbol equations. The first pair is derived straightforwardly from the weak formulation of the boundary value problem (39) of $p^{ \pm}$. It involves one integral in the $(x, y)$-variables and two in the $\xi$-variable, so it turns out to be prohibitive in terms of computation cost, and we have not implemented it. The second pair does not suffer from this drawback, indeed it requires only two integrals, but it is unsymmetrical.

First weak formulation. It states: Find $\mu^{N \pm}$ the symbol, related to (52) an approximate kernel, satisfying

$$
\begin{gathered}
\int_{\Omega^{ \pm}}\left\langle K^{ \pm} D_{-\theta^{ \pm}}^{T} \mu^{N \pm}, e^{\mp \theta^{ \pm}(x-y)}\right\rangle\left\langle K^{ \pm} D_{-\theta^{ \pm}}^{T} \eta^{N \pm}, e^{\mp \theta^{ \pm}(x-y)}\right\rangle d y d x \\
=\int_{\Omega^{ \pm}} q\left\langle\eta^{N \pm}, e^{\mp \theta^{ \pm}(x-y)}\right\rangle d y d x,
\end{gathered}
$$

for all symbols $\eta^{N \pm}$ related to any kernel

$$
v^{N \pm}(x, y)=\sum_{k=0}^{N_{1}} \sum_{\ell=0}^{N_{2}}(1-x) x^{k+1}\left(1-e^{ \pm y}\right)\left(e^{ \pm 1}-e^{ \pm y}\right) e^{ \pm \ell y} v_{k \ell}^{ \pm} .
$$

Its derivation starts from the weak formulation of (39): Find $p^{ \pm} \in H_{\Gamma_{x}^{ \pm} \cup \Gamma_{y}^{ \pm}}^{1}\left(\Omega^{ \pm}\right)=$ $\left\{v^{ \pm} \in H^{1}\left(\Omega^{ \pm}\right) \mid v^{ \pm}=0\right.$ on $\left.\Gamma_{x}^{ \pm} \cup \Gamma_{y}^{ \pm}\right\}$,

$$
\int_{\Omega^{ \pm}} \nabla p^{ \pm} . \nabla v^{ \pm} d y d x=\int_{\Omega^{ \pm}} q v^{ \pm} d y d x \text { for all } v^{ \pm} \in H_{\Gamma_{x}^{ \pm} \cup \Gamma_{y}^{ \pm}}^{1}\left(\Omega^{ \pm}\right) .
$$


Applying the Galerkin method in the base generating (52) yields

$$
\int_{\Omega^{ \pm}} \nabla p^{N \pm} \cdot \nabla v^{N \pm} d y d x=\int_{\Omega^{ \pm}} q v^{N \pm} d y d x \text { for all } v^{N \pm} .
$$

The conclusion follows after stating that the complex symbols associated with $\nabla p^{N \pm}$ and $\nabla v^{N \pm}$ are $K^{ \pm} D_{-\theta^{ \pm}}^{T} \mu^{ \pm}$and $K^{ \pm} D_{-\theta^{ \pm}}^{T} \eta^{N \pm}$ in other terms,

$$
\begin{aligned}
& \nabla\left\langle\mu^{N \pm}(x, .), e^{\mp \theta^{ \pm}(x-y)}\right\rangle=\left\langle K^{ \pm} D_{-\theta^{ \pm}}^{T} \mu^{N \pm}(x, .), e^{\mp \theta^{ \pm}(x-y)}\right\rangle, \\
& \nabla\left\langle\eta^{N \pm}(x, .), e^{\mp \theta^{ \pm}(x-y)}\right\rangle=\left\langle K^{ \pm} D_{-\theta^{ \pm}}^{T} \eta^{N \pm}, e^{\mp \theta^{ \pm}(x-y)}\right\rangle .
\end{aligned}
$$

Second weak formulation. For $h^{+}(x)=x, h^{-}(x)=1-x$, and a function $y \mapsto w(y) \in L^{1}\left(0, h^{ \pm}(x)\right)$, we define the linear operator

$$
L^{ \pm}(w)=\int_{0}^{h^{ \pm}(x)} w e^{-\theta^{ \pm} y} d y
$$

The bases for the approximation of $\mu^{ \pm}$, are $\left(\varphi_{k}^{1}(x) \zeta_{\ell}^{ \pm}(x, \xi)\right)_{k=1, . ., N_{1}, \ell=1, . ., N_{2}}$, the symbol solutions, with

$$
\varphi_{k}^{1}(x)=(1-x) x^{k+1}, \zeta_{\ell}^{ \pm}(x, \xi)=\mp \frac{\left(\theta^{ \pm}(\xi)\right)^{\prime}}{2 i \pi}\left(\frac{1}{\ell+1-\theta^{ \pm}(\xi)}-\frac{e^{-h^{ \pm}(x)}}{\ell-\theta^{ \pm}(\xi)}\right)
$$

with singularities to the left of the imaginary axis, so the contours $\theta^{ \pm}$are chosen as in (53) or (54) to strictly enlace $\mathbb{R}^{+}$. The modified impulse responses $\widetilde{q}^{ \pm}$are approximated in the $y$-direction only,

$$
\widetilde{q}^{N_{2} \pm}(x, y)=\sum_{\ell=0}^{N_{2}} \varphi_{\ell}^{2 \pm}(x, y) q_{\ell}^{N_{2}}(x)
$$

by $L^{2}\left(0, h^{ \pm}(x)\right)$-projection in the bases

$$
\varphi_{\ell}^{2 \pm}(x, y)=\left(e^{-y}-e^{-h^{ \pm}(x)}\right) e^{-\ell y} \text { for } \ell=1, \ldots, N_{2} .
$$

Their corresponding symbols are denoted by $\nu^{N_{2} \pm}(x, \xi)$. The test functions are chosen in different bases with respect to $y$,

$$
\widetilde{v}^{N \pm}(x, y)=\sum_{k=0, \ell=0}^{N_{1}, N_{2}} \varphi_{k}^{1}(x) \varphi_{\ell}^{3 \pm}(x, y) v_{k \ell}^{ \pm} \text {with } \varphi_{\ell}^{3 \pm}(x, y)=\left(e^{y}-e^{h^{ \pm}(x)}\right) e^{\ell y} .
$$

Accordingly, the resulting Petrov-Galerkin method states: Find

$$
\mu^{N \pm}(x, \xi)=\sum_{k=0, \ell=0}^{N_{1}, N_{2}} \varphi_{k}^{1}(x) \zeta_{\ell}^{ \pm}(x, \xi) \mu_{k \ell}^{ \pm}
$$

the solution to

$$
\begin{gathered}
\int_{\omega}\left\langle K^{ \pm} D_{-\theta^{ \pm}}^{T} \mu^{N \pm}, K^{ \pm} L^{ \pm}\left(\nabla \widetilde{v}^{N \pm}\right)\right\rangle d x \\
=\int_{\omega}\left\langle\nu^{N \pm}, L^{ \pm}\left(\widetilde{v}^{N \pm}\right)\right\rangle d x \text { for all } \widetilde{v}^{N \pm}
\end{gathered}
$$


To establish it, we restart from (56), we apply the change of variables $\phi^{ \pm}$in the integrals, and we approximate $\widetilde{p}^{ \pm}$, the modified impulse responses, with polynomials in $x$ and with negative exponential in $y$,

$$
\widetilde{p}^{N \pm}(x, y)=\sum_{k=0}^{N_{1}}(1-x) x^{k+1} \sum_{\ell=0}^{N_{2}}\left(e^{-y}-e^{-h(x)}\right) e^{-\ell y} .
$$

The impulse responses $\widetilde{q}^{ \pm}$are replaced by their approximations (58). We notice that the principle used for these approximations does not exactly match the one used in the first weak formulation, but it follows the same lines in using negative exponential polynomials in $y$ for impulse responses. It yields the above expression of $\mu^{N \pm}$. Now, we observe that

$$
\left(\nabla p^{N \pm}\right) \circ \phi^{ \pm}= \pm\left\langle K^{ \pm} D_{-\theta^{ \pm}}^{T} \mu^{N \pm}, e^{-\theta^{ \pm} y}\right\rangle \text { and } q^{N_{2} \pm} \circ \phi^{ \pm}= \pm\left\langle\nu^{N_{2} \pm}, e^{-\theta^{ \pm} y}\right\rangle,
$$

so we readily get the desired conclusion,

$$
\begin{gathered}
\pm \int_{\omega}\left\langle K^{ \pm} D_{-\theta^{ \pm}}^{T} \mu^{N \pm}, K^{ \pm} \int_{0}^{h^{ \pm}(x)} \nabla \widetilde{v}^{N \pm} e^{-\theta^{ \pm} y} d y\right\rangle d x \\
= \pm \int_{\omega}\left\langle\nu^{N_{2} \pm}, \int_{0}^{h^{ \pm}(x)} \widetilde{v}^{N \pm} e^{-\theta^{ \pm} y} d y\right\rangle d x .
\end{gathered}
$$

We observe that

$$
\begin{aligned}
& L^{ \pm}\left(\widetilde{v}^{ \pm}\right)(x, \xi) \\
& \quad=\sum_{k=0}^{N_{1}}(1-x) x^{k+1} \sum_{\ell=0}^{N_{2}}\left(\frac{e^{\left(\ell+1-\theta^{ \pm}(\xi)\right) h^{ \pm}(x)}-1}{\ell+1-\theta^{ \pm}(\xi)}-\frac{e^{h^{ \pm}(x)}\left(e^{\left(\ell-\theta^{ \pm}(\xi)\right) h^{ \pm}(x)}-1\right)}{\ell-\theta^{ \pm}(\xi)}\right) v_{k l}^{ \pm},
\end{aligned}
$$

so the choice of the base for $\widetilde{v}^{ \pm}$has been made so that the poles of

$$
\lambda \mapsto \sum_{k=0}^{N_{1}}(1-x) x^{k+1} \sum_{\ell=0}^{N_{2}}\left(\frac{e^{(\ell+1+\lambda) h^{ \pm}(x)}-1}{\ell+1+\lambda}-\frac{e^{h^{ \pm}(x)}\left(e^{(\ell+\lambda) h^{ \pm}(x)}-1\right)}{\ell+\lambda}\right) v_{k l}^{ \pm},
$$

are in the negative real axis like those of the symbols $\mu^{N \pm}$.

To conduct the direct discretizations of $\mu^{N+}$ we have used the second weak formulation, and the same elements as in Subsection 5.1] namely the same contours, the same discretization of $\psi$, and the same approximation of diffusive realization. The data are also kept the same, i.e., the same kernel $q$, and the same input variable $u$. The related approximation results (Symbol) are also reported in Figure 2. For piecewise constant interpolation (C), the error decay rates are the same as for the method based on kernel calculation. This is also true for piecewise linear interpolation (L) until the number of spatial discretization points reaches $1 / h \approx 80$. Beyond this limit the rate of convergence slows down due to ill conditioning of the linear system.

\section{Appendix}

We state some technical results used in Section 4.2 for real diffusive symbol calculation. We state them in the general framework of distributions of $\mathcal{D}^{\prime}(\mathbb{R})$ but we apply them to distributions of $\mathcal{D}_{+}^{\prime}$ with support is in $\mathbb{R}^{+}$. 
Lemma 23. (i) For $p, n \in \mathbb{N}$,

$$
\begin{aligned}
\xi^{p} \delta^{(n)} & =(-1)^{p} n(n-1) \cdots(n-p+1) \delta^{(n-p)} & & \text { for } p \leq n, \\
& =0 & & \text { for } p \geq n+1 .
\end{aligned}
$$

In particular,

$$
\xi \delta^{(n)}=-n \delta^{(n-1)} \text { for } n \geq 1 \text { and } \xi^{2} \delta^{(n)}=n(n-1) \delta^{(n-2)} \text { for } n \geq 2 .
$$

(ii) For $p, n \in \mathbb{N}$ and $x>0$,

$$
\begin{aligned}
\xi^{p} e^{-\xi x} \delta^{(n)} & =(-1)^{p} \sum_{k=p}^{n} \frac{x^{k-p} n !}{(k-p) !(n-k) !} \delta^{(n-k)} & & \text { for } p \leq n, \\
& =0 & & \text { for } p \geq n+1 .
\end{aligned}
$$

In particular,

$$
\begin{gathered}
\xi e^{-\xi x} \delta^{(n)}(\xi)=-\sum_{k=1}^{n} \frac{x^{k-1} n !}{(k-1) !(n-k) !} \delta^{(n-k)}(\xi) \text { for } n \geq 1, \\
\xi^{2} e^{-\xi x} \delta^{(n)}(\xi)=\sum_{k=2}^{n} \frac{x^{k-2} n !}{(k-2) !(n-k) !} \delta^{(n-k)}(\xi) \text { for } n \geq 2 .
\end{gathered}
$$

Proof. The first point is very classical. We derive only the second:

$$
\begin{gathered}
\left\langle\xi^{p} e^{-\xi x} \delta^{(n)}, \varphi\right\rangle=(-1)^{n}\left\langle\delta,\left(e^{-\xi x} \xi^{p} \varphi\right)^{(n)}\right\rangle=(-1)^{n} \sum_{k=0}^{n} C_{n}^{k}\left\langle\delta,\left(e^{-\xi x} \xi^{p}\right)^{(k)} \varphi^{(n-k)}\right\rangle \\
=(-1)^{n}\left(\sum_{k=0}^{p} \sum_{l=0}^{k}+\sum_{k=p+1}^{n} \sum_{l=0}^{p}\right) C_{k}^{l} C_{n}^{k}(-x)^{k-l} p(p-1) \\
\cdots(p-l+1)\left\langle\delta, \xi^{p-l} e^{-\xi x} \varphi^{(n-k)}\right\rangle .
\end{gathered}
$$

We observe that $\left\langle\delta, \xi^{p-l} e^{-\xi x} \varphi^{(n-k)}\right\rangle=0$ for $l<p$ and $=\varphi^{(n-k)}(0)$ for $l=p$, so it remains

$$
=(-1)^{n} p ! C_{n}^{p}\left\langle\delta, e^{-\xi x} \varphi^{(n-p)}\right\rangle+p ! \sum_{k=p+1}^{n}(-1)^{n+k-p} C_{k}^{l} C_{n}^{k} x^{k-p}\left\langle\delta, e^{-\xi x} \varphi^{(n-k)}\right\rangle .
$$

After simplifications,

$$
=\frac{(-1)^{p} n !}{(n-p) !}\left\langle\delta^{(n-p)}, \varphi\right\rangle+(-1)^{p} \sum_{k=p+1}^{n} \frac{x^{k-p} n !}{(k-p) !(n-k) !}\left\langle\delta^{(n-k)}, \varphi\right\rangle .
$$

Finally,

$$
\left\langle\xi^{p} e^{-\xi x} \delta^{(n)}, \varphi\right\rangle=(-1)^{p} \sum_{k=p}^{n} \frac{x^{k-p} n !}{(k-p) !(n-k) !}\left\langle\delta^{(n-k)}, \varphi\right\rangle,
$$

which is (64), from which follow (65), 666).

\section{ACKNOWLEDGMENTS}

We are grateful to Boris Andrianov for his help with degenerate differential equations in distribution spaces. 


\section{REFERENCES}

[1] B. Bamieh, F. Paganini, and M.A. Dahleh, Distributed control of spatially invariant systems, IEEE Transactions on Automatic Control 47 (2002), no. 7, 1091-1107. MR1911479 (2003d:93044)

[2] P. Bidan, T. Lebey, G. Montseny, C. Neascu, and J. Saint-Michel, Transient voltage distribution in inverter fed motor windings: Experimental study and modeling, IEEE Transactions on Power Electronics 16 (2001), no. 1, 92-100.

[3] P. Bidan, T. Lebey, and C. Neacsu, Development of a new off-line test procedure for low voltage rotating machines fed by adjustable speed drives (ASD), IEEE Transactions on Dielectrics and Electrical Insulation 10 (2003), no. 1, 168-175.

[4] C. Casenave and E. Montseny, Time-local dissipative formulation and stable numerical schemes for a class of integrodifferential wave equations, SIAM J. Appl. Math. 68 (2008), no. 6, 1763-1782. MR2424962 (2009g:45017)

[5] E. Cuesta, C. Lubich, and C. Palencia, Convolution quadrature time discretization of fractional diffusion-wave equations, Math. Comp. 75 (2006), no. 254, 673-696. MR2196986 (2006j:65404)

[6] R. D'Andrea and G. E. Dullerud, Distributed control design for spatially interconnected systems, IEEE Trans. Automat. Control 48 (2003), no. 9, 1478-1495. MR2000106|(2004f:93042)

[7] I. P. Gavrilyuk and V. L. Makarov, Exponentially convergent parallel discretization methods for the first order evolution equations, Comput. Methods Appl. Math. 1 (2001), no. 4, 333355. MR1892950 (2003f:65174)

[8] __ Exponentially convergent algorithms for the operator exponential with applications to inhomogeneous problems in Banach spaces, SIAM J. Numer. Anal. 43 (2005), no. 5, 21442171. MR2192335 (2006m:65100)

[9] G. Goavec-Merou, Y. Yakoubi, R. Couturier, M. Lenczner, and J.-M. Friedt, Fpga implementation of diffusive realizations for a distributed control operator, dMEMS 2010, Besançon, France - June 28-29th 2010.

[10] T. Hélie and D. Matignon, Diffusive representations for the analysis and simulation of flared acoustic pipes with visco-thermal losses, Math. Models Methods Appl. Sci. 16 (2006), no. 4, 503-536. MR2218212 (2006k:76123)

[11] T. Hélie, D. Matignon, and R. Mignot, Criterion design for optimizing low-cost approximations of infinite-dimensional systems: towards efficient real-time simulation, Int. J. Tomogr. Stat. 7 (2007), no. F07, 13-18. MR.2393796

[12] C. Langbort and R. D'Andrea, Distributed control of spatially reversible interconnected systems with boundary conditions, SIAM J. Control Optim. 44 (2005), no. 1, 1-28. MR2176664 (2006h:93006)

[13] L. Laudebat, P. Bidan, and G. Montseny, Modeling and optimal identification of pseudodifferential electrical dynamics by means of diffusive representation - Part 1: Modeling, IEEE Transactions on Circuits and Systems I-Regular Papers 51 (2004), no. 9, 1801-1813. MR2090345 (2005e:94302)

[14] M. Lenczner and G. Montseny, Diffusive realization of operator solutions of certain operational partial differential equations, C. R. Math. Acad. Sci. Paris 341 (2005), no. 12, 737-740. MR2188868 (2006f:93053)

[15] M. Lenczner and Y. Yakoubi, Semi-decentralized approximation of optimal control for partial differential equations in bounded domains, Comptes Rendus Mécanique 337 (2009), no. 4, 245-250.

[16] D. Levadoux and G. Montseny, Diffusive realization of the impedance operator on circular boundary for $2 D$ wave equation, Mathematical and numerical aspects of wave propagationWAVES 2003, Springer, Berlin, 2003, pp. 136-141. MR2077989

[17] M. López-Fernández, C. Lubich, C. Palencia, and A. Schädle, Fast Runge-Kutta approximation of inhomogeneous parabolic equations, Numer. Math. 102 (2005), no. 2, 277-291. MR2206466 (2007c:65071)

[18] M. López-Fernández, C. Lubich, and A. Schädle, Adaptive, fast, and oblivious convolution in evolution equations with memory, SIAM J. Sci. Comput. 30 (2008), no. 2, 1015-1037. MR.2385897 (2008m:65363) 
[19] M. López-Fernández and C. Palencia, On the numerical inversion of the Laplace transform of certain holomorphic mappings, Appl. Numer. Math. 51 (2004), no. 2-3, 289-303. MR2091405 (2005e:65210)

[20] C. Lubich, Convolution quadrature revisited, BIT 44 (2004), no. 3, 503-514. MR 2106013 (2005f:65175)

[21] C. Lubich and A. Schädle, Fast convolution for nonreflecting boundary conditions, SIAM J. Sci. Comput. 24 (2002), no. 1, 161-182. MR1924419 (2003h:44007)

[22] D. Matignon and C. Prieur, Asymptotic stability of linear conservative systems when coupled with diffusive systems, ESAIM Control Optim. Calc. Var. 11 (2005), no. 3, 487-507. MR.2148855 (2006f:93054)

[23] W. McLean and V. Thomée, Time discretization of an evolution equation via Laplace transforms, IMA J. Numer. Anal. 24 (2004), no. 3, 439-463. MR2068831 (2005d:47072)

[24] G Montseny, Simple approach to approximation and dynamical realization of pseudodifferential time operators such as fractional ones, IEEE Transactions on Circuits and Systems II-Express Briefs 51 (2004), no. 11, 613-618.

[25] G. Montseny, Représentation diffusive, Hermès-Sciences, 2005.

[26] A. Schädle, M. López-Fernández, and C. Lubich, Fast and oblivious convolution quadrature, SIAM J. Sci. Comput. 28 (2006), no. 2, 421-438. MR2231714 (2007b:65142)

[27] L. Schwartz, Méthodes mathématiques pour les sciences physiques, Enseignement des Sciences, Hermann, Paris, 1961. MR0143360 (26:919)

[28] D. Sheen, I. H. Sloan, and V. Thomée, A parallel method for time discretization of parabolic equations based on Laplace transformation and quadrature, IMA J. Numer. Anal. 23 (2003), no. 2, 269-299. MR 1975267 (2004b:65161)

[29] A. Talbot, The accurate numerical inversion of Laplace transforms, J. Inst. Math. Appl. 23 (1979), no. 1, 97-120. MR.526286 (80c:65244)

[30] L. N. Trefethen, J. A. C. Weideman, and T. Schmelzer, Talbot quadratures and rational approximations, BIT 46 (2006), no. 3, 653-670. MR.2265580(2007k:41033)

[31] C. Wagschal, Fonctions holomorphes, Equations différentielles, Hermann, Paris, 2003.

[32] J. A. C. Weideman and L. N. Trefethen, Parabolic and hyperbolic contours for computing the Bromwich integral, Math. Comp. 76 (2007), no. 259, 1341-1356. MR.2299777(2008c:65072)

[33] K. Yosida, Functional analysis, Classics in Mathematics, Springer-Verlag, 1995, Reprint of the sixth (1980) edition. MR.1336382 (96a:46001)

Femto-St Institute, Time-Frequency 26, Rue de l'Epitaphe, 25030 Besançon, France -And- UTBM, 90010 Belfort Cedex, France

E-mail address: michel.lenczner@utbm.fr

LAAS-CNRS 7, avenue du Colonel Roche 31077 Toulouse Cedex 4, France

E-mail address: montseny@laas.fr

UPMC Univ Paris 06, Laboratoire Jacques-Louis Lions, F-75005, Paris Cedex, France E-mail address: yyakoubi@ann.jussieu.fr 